\title{
A fourth-order compact scheme for the Helmholtz equation: Alpha-interpolation of FEM and FDM stencils
}

\author{
Prashanth Nadukandi* ${ }^{\dagger}$, Eugenio Oñate and Julio Garcia \\ International Center for Numerical Methods in Engineering (CIMNE), Universitat Politecnica de Catalunya \\ (UPC), Campus Nord, Edifici C1, Gran Capitan s/n, 08034 Barcelona, Spain
}

\begin{abstract}
SUMMARY
We propose a fourth-order compact scheme on structured meshes for the Helmholtz equation given by $R(\phi):=f(\mathbf{x})+\Delta \phi+\xi^{2} \phi=0$. The scheme consists of taking the alpha-interpolation of the Galerkin finite element method and the classical central finite difference method. In 1D, this scheme is identical to the alpha-interpolation method (J. Comput. Appl. Math. 1982; 8(1):15-19) and in 2D making the choice $\alpha=0.5$ we recover the generalized fourth-order compact Padé approximation (J. Comput. Phys. 1995; 119:252-270; Comput. Meth. Appl. Mech. Engrg 1998; 163:343-358) (therein using the parameter $\gamma=2$ ). We follow (SIAM Rev. 2000; 42(3):451-484; Comput. Meth. Appl. Mech. Engrg 1995; 128:325-359) for the analysis of this scheme and its performance on square meshes is compared with that of the quasi-stabilized FEM (Comput. Meth. Appl. Mech. Engrg 1995; 128:325-359). In particular, we show that the relative phase error of the numerical solution and the local truncation error of this scheme for plane wave solutions diminish at the rate $O\left((\xi \ell)^{4}\right)$, where $\xi$, $\ell$ represent the wavenumber and the mesh size, respectively. An expression for the parameter $\alpha$ is given that minimizes the maximum relative phase error in a sense that will be explained in Section 4.5. Convergence studies of the error in the $L^{2}$ norm, the $H^{1}$ semi-norm and the $l^{\infty}$ Euclidean norm are done and the pollution effect is found to be small. Copyright (C) 2010 John Wiley \& Sons, Ltd.
\end{abstract}

Received 29 December 2009; Revised 6 August 2010; Accepted 12 August 2010

KEY WORDS: Helmholtz equation; alpha-interpolation of FEM and FDM; compact stencils; dispersion analysis

\section{INTRODUCTION}

In this paper, we study the Helmholtz equation given by $R(\phi):=f(\mathbf{x})+\Delta \phi+\xi^{2} \phi=0$ and subjected to Dirichlet boundary conditions. The solution $\phi$ to this equation is oscillatory and $\xi$ is the wave number (spatial frequency) of $\phi$. If $\lambda_{m}$ is an eigenvalue of the operator $-\Delta$, then for $\xi \neq \sqrt{\lambda_{m}}$ the problem has a unique solution. On the contrary, i.e. for $\xi=\sqrt{\lambda_{m}}$ the problem is indefinite. In this case if the equation and the Dirichlet boundary conditions are homogeneous, then we end up in a differential eigenvalue problem. It follows that the solution is not unique and can be represented as a scalar multiple of the eigenfunction corresponding to each eigenvalue. Let $\lambda_{m}^{h}$ represent an eigenvalue of the problem after any appropriate discretization. Unlike the set of eigenvalues $\left\{\lambda_{m}\right\}$ that is infinite, the set $\left\{\lambda_{m}^{h}\right\}$ is finite and its dimension is equal to that of the discrete space. Thus, when the wave number $\xi \rightarrow \sqrt{\lambda_{m}^{h}}$, the discrete problem tends to be indefinite. This case is usually referred to as the case of degeneracy and here the discrete problem is ill-conditioned.

\footnotetext{
${ }^{*}$ Correspondence to: Prashanth Nadukandi, International Center for Numerical Methods in Engineering (CIMNE), Universitat Politecnica de Catalunya (UPC), Campus Nord, Edifici C1, Gran Capitan s/n, 08034 Barcelona, Spain.

†E-mail: npras@cimne.upc.edu
} 
As the current problem admits a variational principle, naturally, discretization methods based on variational formulations viz. the Galerkin and the Trefftz-Galerkin-type methods have been preferred to other methods. The Galerkin-type methods are domain-based wherein the integral statement involves only the weak form of the governing differential equation and the sub-space of test-functions are assumed to satisfy a priori the kinematic compatibility and essential boundary conditions. The Trefftz-Galerkin-type methods are boundary-based and are formulated using the reciprocal principle wherein the integral statement involves only the kinematic compatibility and essential boundary conditions of the problem and the sub-space of test-functions are assumed to satisfy a priori the governing differential equation $[1,2]$.

In the context of the Galerkin-type methods, the finite element method (FEM) is a powerful technique to systematically generate subspaces of test-functions (classically piecewise polynomial spaces). Some of the earlier works on the use of FEM for the numerical solution of the Helmholtz equation can be found in [3-10] and the references cited therein. In [5, 7] error estimates were given for the asymptotic $\left(\xi^{2} \ell\right.$ assumed sufficiently small) and pre-asymptotic ( $\xi \ell$ assumed sufficiently small) cases, respectively. It was shown that for the discrete problem the $\mathrm{LBB}^{+}$constant can be expressed as $\gamma^{h}=\min \left\{\left|\lambda_{m}^{h}-\xi^{2}\right| / \lambda_{m}^{h}\right\}$ [6]. Thus, for the continuous problem (visualized as $\ell \rightarrow 0$ ) the LBB constant can be expressed as $\gamma=\min \left\{\left|\lambda_{m}-\xi^{2}\right| / \lambda_{m}\right\}$, which in an average sense implies that $\gamma$ is inversely proportional to the wavenumber $\xi$, i.e. $\gamma \propto \xi^{-1}[6,7]$. Thus for high wavenumbers and for the case of degeneracy $\left(\xi \rightarrow \sqrt{\lambda_{m}^{h}}\right)$, the LBB constant for the discrete problem tends to be small which in turn leads to a loss of stability. The loss of stability with respect to an increase in the wavenumber $\xi$ is called the pollution effect, which is impossible to avoid completely $[9,10]$. Nevertheless, the pollution effect can be controlled unlike the loss of stability for the case of degeneracy where it is Several stabilizatio The Galerkin least-s [11], the extension was also studied. convection-diffusio Following the framework of the generalized fitite
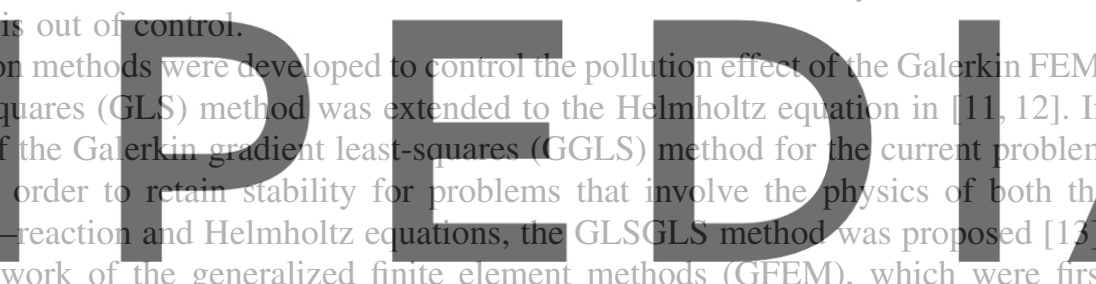

introduced in [14] in a variational setting, the quasi-stabilized FEM (OSFEM) was proposed in

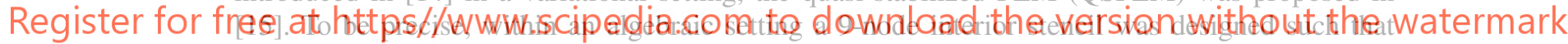

the pollution effect is asymptotically minimal, thus leading to minimal phase error for arbitrary wave direction in 2D. The partition of unity method (PUM) was proposed in [16, 17] by which conforming subspaces of higher regularity can be generated out of a set of local approximation spaces. These local approximation spaces could be designed to include a priori knowledge about the local behavior of the solution. Recently, following the framework of PUM, a locally enriched FEM was proposed in [18] wherein it was shown that the Bessel functions of the first kind could be used to enrich the finite element space instead of the plane waves (as is done in PUM). Another stabilization approach consists of enriching the classical finite element spaces by bubble functions. Following this line the residual-free bubbles (RFB) method was extended to the Helmholtz equation in [19]. Another bubble-based method is the nearly optimal PetrovGalerkin method (NOPG) presented in [20]. A comparison of the RFB and NOPG methods for the Helmholtz equation was done in [21]. Recently, another GFEM was proposed in [22] in which the classical FEM is enriched by plane waves pasted into the finite element basis at each mesh vertex by the PUM. Also, this method allows the use of Cartesian meshes that may overlap the boundaries of the problem domain. This GFEM was further developed in [23] wherein the effects of using alternative handbook functions and mesh types are addressed. Based on the variational multiscale (VMS) method, several stabilization methods were proposed, viz. the sub-grid FEM [24], the two sub-grid scale (SGS) models presented in [25], the residual-based FEM (RBFEM)

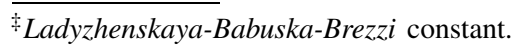


[26] and more recently, the algebraic subgrid FEM (ASGS) [27] and the SGS-GSGS method [28]. Following the more general VMS method wherein the subscales are not modeled as bubbles, the RBFEM method also includes the residuals on the inter-element boundaries while retaining the sparsity of the Galerkin method. As in the GLSGLS method, the SGS-GSGS method attempts to stabilize the advection-diffusion-reaction/production problem and is designed to be nodally exact in 1D. Within the framework of the discontinuous Galerkin (DG) method, the discontinuous enrichment method (DEM) was proposed $[29,30]$ wherein the classical finite element spaces are enriched (as in bubble-based methods) via a set of local approximation spaces (as in the PUM-based methods). In the DEM, the continuity of the enrichment across element boundaries is enforced weakly by Lagrange multipliers (unlike the PUM-based methods) and it need not vanish at the element boundaries (unlike the bubble-based methods). Another DG method is presented in [31] wherein the continuity of the finite element spaces across the element edges is relaxed and weakly enforced via two penalty parameters corresponding to possible jumps of the solution field and its gradient. These penalty parameters are designed to minimize the pollution error. Following the ideas of the former DG method [31], another discontinuous FEM was proposed in [32] (therein called as the DGB method). In the DGB method, the classical finite element spaces are enriched via bubbles that are allowed to be discontinuous across subgrid patches. Following the DG method in [31], the continuity of the bubble spaces across interior patch boundaries is enforced weakly via two penalty parameters corresponding to possible jumps of the solution field and its gradient. Again, these penalty parameters are designed to minimize the pollution error. Nodally exact Ritz discretization of the 1D diffusion-absorption/production equations via variational finite calculus (FIC) and modified equation methods using a single stabilization parameter were presented in [33]. The Galerkin-projected residual (GPR) method for the Helmholtz equa-

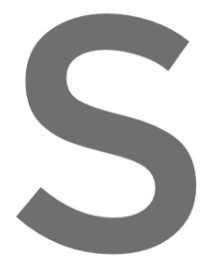
tion was presented done in [35].

Owing to the abs method. Neverthele best one can get on using bilinear finite element was proposed in
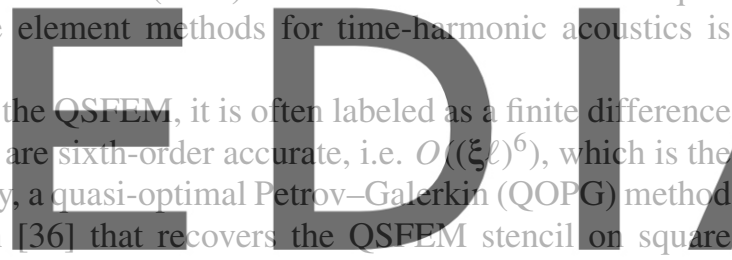

meshes. In the QOPG method, the Galerkin FEM weights are perturbed by a quadratic bubble

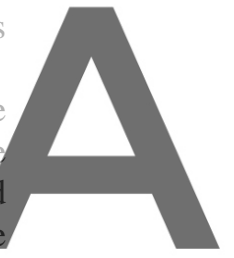

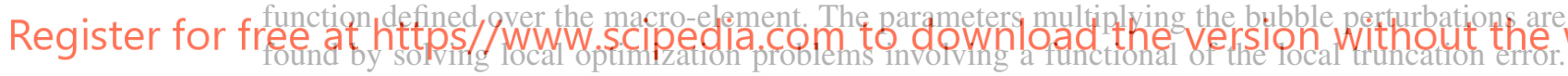

watermark

Later, following this line, a quasi-optimal finite difference method on generic unstructured meshes was proposed in [37].

Within the framework of the finite difference methods, several fourth-order compact schemes obtained through a generalization of the fourth-order Padé approximation were studied in $[38,39]$. Following this line, two new finite difference methods (FDMs) were proposed in [40] that achieve sixth- and eighth-order accuracy, respectively, using a five-point (and hence noncompact) stencil in 1D. In [41], a new FDM with improved accuracy was proposed by modifying the central difference scheme (i.e. the classical FDM) by replacing the weight multiplying the central node with an optimal expression that used Bessel's function of the first kind. The FLAME method was proposed in [42] that exploits the use of local approximating functions to define higher-order finite difference schemes on a chosen stencil. In particular, on a compact stencil a sixth-order accurate scheme for the Helmholtz equation can be derived using the FLAME method. Sixth-order accurate FD schemes on a compact stencil for the Helmholtz equation were proposed in [43-45]. An alternate approach to derive FDMs is the global method of differential quadrature (DQ) [46]. Following this line, a polynomial-based DQ and a Fourier expansion-based DQ were derived for the Helmholtz equation in [47]. As higher-order polynomial or sinusoidal interpolation functions are employed, these methods reduce the restriction on the mesh resolution to the Nyquist limits, i.e. the rule of thumb for these methods is to provide at least two elements per wavelength.

Some of the earlier works in the context of the Trefftz-Galerkin-type methods could be found in the seminal papers [48-51]. A treatise on Trefftz-type methods can be found in [52,53]. Specifically, the Trefftz-type methods were used for the Helmholtz equation in [54-58]. The case of 
degeneracy, i.e. $\xi \rightarrow \lambda_{m}^{h}$, is considered for the first time in [58] and the error asymptote of the solution by the Trefftz method is given.

In this paper, we present some observations and related dispersion analysis of a domain-based fourth-order compact scheme for the Helmholtz equation. In other words, the phase error of the numerical solution and the local truncation error of this scheme for plane wave solutions diminish at the rate $O\left((\xi \ell)^{4}\right)$. The focus is on the approximation of the Helmholtz equation in the interior of the domain using compact stencils. The scheme consists of taking the alpha-interpolation of the Galerkin FEM and the classical FDM. This scheme has its origins in an old idea that marks the point of departure: to replace the consistent mass matrix $\mathbf{M}$ in the Galerkin FEM by a higherorder mass matrix $\mathbf{M}^{0.5}:=\left(\mathbf{M}+\mathbf{M}_{\mathrm{L}}\right) / 2$, where $\mathbf{M}_{\mathbf{L}}$ is the lumped mass matrix. This idea was proposed independently for eigenvalue problems by Goudreau [59], Goudreau and Taylor [60] and Ishihara [61]. In the later work, the matrix $\mathbf{M}^{0.5}$ was denominated as the mixed-mass (MM) matrix and as a concluding remark the generalized mixed mass (GMM) scheme was proposed as an extension to the MM scheme where an $\alpha$-interpolation of the mass matrices is done, i.e. $\mathbf{M}^{\alpha}:=\alpha \mathbf{M}+(1-\alpha) \mathbf{M}_{\mathrm{L}}$. This GMM scheme was later bantized as the alpha-internolation method (AIM) [62] and was extended to the hollow waveguide analysis in [63] and the Schrodinger equation in [64]. For the simple 1D case, our scheme mimics the AIM and in 2D making the choice $\alpha=0.5$ we recover the generalized fourth-order compact Padé approximation [38,39] (therein using the parameter $\gamma=2$ ).

The paper is organized as follows. In Section 2, we present the statement of the Helmholtz equation viewed as a diffusion-production problem. This is done only to facilitate future assimilation of ideas toward a generic method that would aim at stabilizing problems that involve the physics of both the convection-diffusion-reaction and Helmholtz equations. In Section 3, we present the analysis of the problem in $1 \mathrm{D}$. The expressions for the numerical solution of our scheme and its relative phase a series expansion approximation proper zones of degeneracy. that results from a two
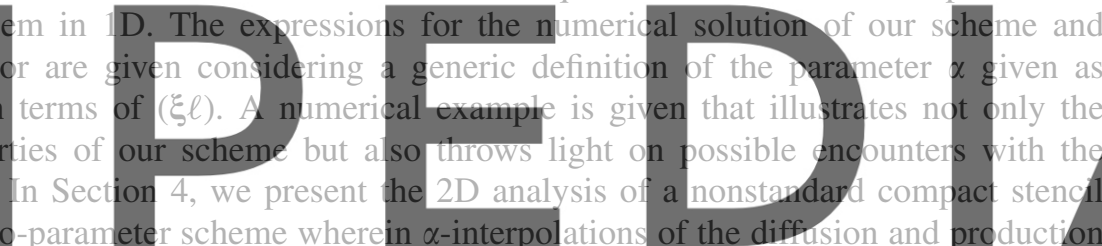

terms are done independently and it can model several methods (including the QSFEM). This

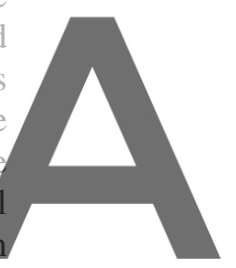

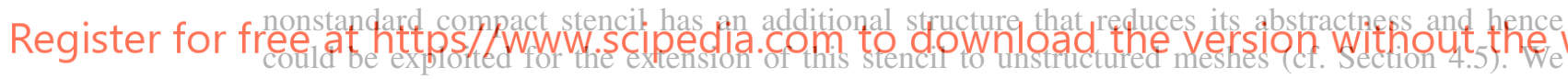
follow $[10,15]$ for the analysis of this stencil and its performance on square meshes is compared with that of the QSFEM [15]. Just like in $1 D$, we try to express the numerical solution of this stencil in 2D considering generic definitions of the parameters given as a series expansion in terms of $(\xi \ell)$. Using this expression for the numerical solution, the expressions for the relative phase and local truncation errors are given. In particular for our scheme, i.e. the $\alpha$-interpolation of the FEM and FDM stencils, an optimal expression for the parameter $\alpha$ is given. The dispersion plots in 2D and related discussion are done in Section 4.6. Some examples are presented in Section 4.7 that illustrate the pollution effect through convergence studies in the $L^{2}$ norm, $H^{1}$ semi-norm and the $l^{\infty}$ Euclidean norms. Finally in Section 5, we remark on the extension of our scheme to unstructured meshes and arrive at some conclusions.

\section{PROBLEM STATEMENT}

The statement of the multidimensional Helmholtz equation subjected to Dirichlet boundary conditions is as follows:

$$
\begin{aligned}
R(\phi):=k \Delta \phi+s \phi+f(\mathbf{x}) & =0 \quad \text { in } \Omega \\
\phi & =\phi^{\mathrm{p}} \quad \text { on } \Gamma_{\mathrm{D}}
\end{aligned}
$$

where $k>0, s>0$ are the diffusion and production coefficients, respectively, $f(\mathbf{x})$ is the source and $\phi^{\mathrm{P}}$ is the prescribed value of $\phi$ at the Dirichlet boundary. When $s<0$, Equation (1a) represents 
the diffusion-reaction problem that models the mass transfer processes with first-order chemical reactions and wherein $s$ represents the reaction coefficient.

The variational statement of the problem (1a) can be expressed as follows: Find $\phi \in V$ such that $\forall w \in V_{0}$ we have

$$
\begin{aligned}
a(w, \phi) & =l(w) \\
a(w, \phi) & :=\int_{\Omega}(k \nabla w \cdot \nabla \phi-s w \phi) \mathrm{d} \Omega \\
l(w) & :=\int_{\Omega} w f(\mathbf{x}) \mathrm{d} \Omega
\end{aligned}
$$

where $V:=\left\{w: w \in H^{1}(\Omega)\right.$ and $w=\phi^{\mathrm{p}}$ on $\left.\Gamma_{\mathrm{D}}\right\}$ and $V_{0}:=\left\{w: w \in H^{1}(\Omega)\right.$ and $w=0$ on $\left.\Gamma_{\mathrm{D}}\right\}$. The statement of the Galerkin method applied to the weak form (2) of the problem is: Find $\phi_{h} \in V^{h}$ such that $\forall w_{h} \in V_{0}^{h}$ we have

$$
a\left(w_{h}, \phi_{h}\right)=l\left(w_{h}\right)
$$

where $V^{h} \subset V$ is a subspace obtained via any appropriate discretization. Discretization of the space by finite elements will lead to the approximation $\phi_{h}=N^{\mathrm{a}} \Phi^{\mathrm{a}}$ and Equation (3) reduces into the following system of equations:

$$
[k \mathbf{D}-s \mathbf{M}] \Phi=\mathbf{f}
$$
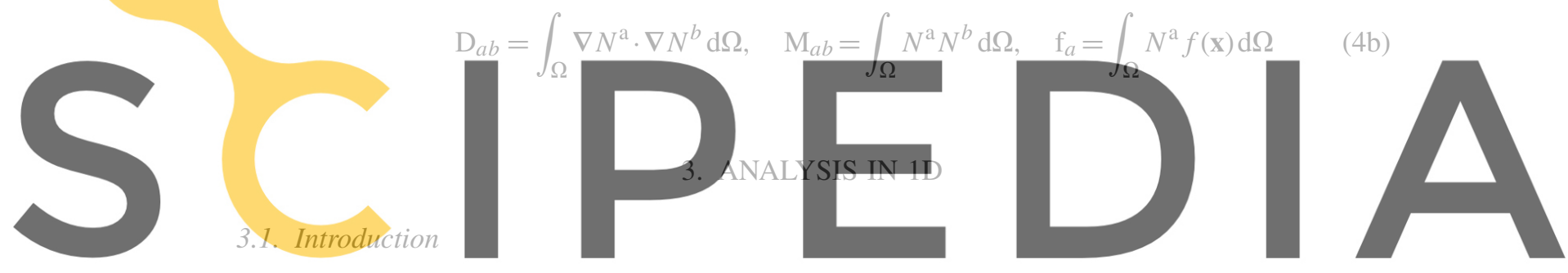

In this section, we study the homogeneous Helmholtz equation in 1D subjected to Dirichlet

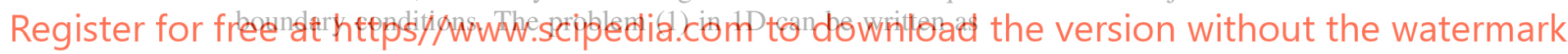

$$
\begin{gathered}
k \frac{\mathrm{d}^{2} \phi}{\mathrm{d} x^{2}}+s \phi=0 \quad \text { in } \Omega \\
\phi(x=0)=\Phi^{1}, \quad \phi(x=L)=\Phi^{\mathrm{r}} \quad \text { on } \Gamma_{\mathrm{D}}
\end{gathered}
$$

where $L$ is the length of the 1D domain and $\Phi^{1}, \Phi^{\mathrm{r}}$ are the Dirichlet boundary data at the left and right domain boundaries, respectively. The solution to Equation (5) when $s>0$ is harmonic and is expressed as:

$$
\phi(x)=\frac{\Phi^{1} \sin \left(\xi_{o} L-\xi_{o} x\right)+\Phi^{\mathrm{r}} \sin \left(\xi_{o} x\right)}{\sin \left(\xi_{o} L\right)}
$$

where $\xi_{o}:=\sqrt{s / k}$ is the angular wave number. We also list the eigenvalues of this problem that can be expressed as $\lambda_{m}:=(m \pi / L)^{2} \forall m \in\{1,2,3, \ldots\}$. The element contributions to the matrices given in Equations (4) using 2-node linear finite elements are

$$
\mathbf{D}^{e}=\frac{1}{\ell}\left[\begin{array}{cc}
1 & -1 \\
-1 & 1
\end{array}\right], \quad \mathbf{M}^{e}=\frac{\ell}{6}\left[\begin{array}{ll}
2 & 1 \\
1 & 2
\end{array}\right]
$$

where $\ell$ is the corresponding element length. If the discretization is uniform, the equation stencil for the problem (5a) corresponding to each interior node can be expressed as follows:

$$
\left(\frac{k}{\ell}\right)\left(-\Phi^{i-1}+2 \Phi^{i}-\Phi^{i+1}\right)-\left(\frac{s \ell}{6}\right)\left(\Phi^{i-1}+4 \Phi^{i}+\Phi^{i+1}\right)=0
$$


If the mass matrix $\mathbf{M}$ is lumped, then the equation stencil corresponding to any interior node can be written as follows:

$$
\left(\frac{k}{\ell}\right)\left(-\Phi^{i-1}+2 \Phi^{i}-\Phi^{i+1}\right)-s \ell \Phi^{i}=0
$$

This is also the stencil we get using the classical FDM. ${ }^{\S}$

\section{2. $\alpha$-interpolation of the Galerkin-FEM and the classical FDM}

Define a free parameter $\alpha$ and consider the $\alpha$-interpolation of the stencils obtained by the Galerkin FEM and the classical FDM methods for the problem (5):

$$
\begin{aligned}
& (1-\alpha)\left[\left(\frac{k}{\ell}\right)\left(-\Phi^{i-1}+2 \Phi^{i}-\Phi^{i+1}\right)-\left(\frac{s \ell}{6}\right)\left(\Phi^{i-1}+4 \Phi^{i}+\Phi^{i+1}\right)\right] \\
& +\alpha\left[\left(\frac{k}{\ell}\right)\left(-\Phi^{i-1}+2 \Phi^{i}-\Phi^{i+1}\right)-s \ell \Phi^{i}\right]=0 \\
& \Rightarrow\left(\begin{array}{c}
k \\
\ell
\end{array}\right)\left(-\Phi^{i-1}+2 \Phi^{i}-\Phi^{i+1}\right)-(1-\alpha)\left(\frac{s \ell}{6}\right)\left(\Phi^{i-1}+4 \Phi^{i}+\Phi^{i+1}\right)-\alpha s \ell \Phi^{i}=0 \\
& \Rightarrow\left(\frac{k}{\ell}-\alpha \frac{s \ell}{6}\right)\left(-\Phi^{i-1}+2 \Phi^{i}-\Phi^{i+1}\right)-\left(\frac{s \ell}{6}\right)\left(\Phi^{i-1}+4 \Phi^{i}+\Phi^{i+1}\right)=0
\end{aligned}
$$

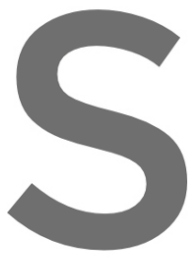

Remark: In 1D, we can arive at the above equations through ar alternative argument: C
the Galerkin FEM method using the $\alpha$ interpolated mass matrix $\mathbf{M}^{\alpha}$. The later rrgument
the AIM. A particular case (taking $\alpha=0.5$ ) is the MM scheme proposed by Ishihara applied
Helmholtz equation [61]. The Mivi matrix ( $\mathbf{M}^{0.5}$ ) was carther refered to as the higher-ord
matrix by Goudreau [59]. In 1I), a stencil equivalent to the MM scheme will be obtaine
the compact fourth-order Padé approximation to problem (5) $[38,39]$.

We can guess that a solution to Equation (10) takes the form $\Phi^{i}:=\phi\left(x_{i}\right)=\exp \left(i \xi^{h} x_{i}\right)$. Substi-

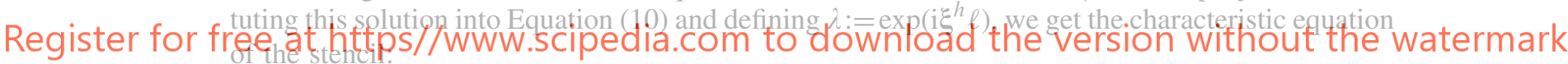

$$
\lambda^{2}-2\left(\frac{6-(2+\alpha) \omega}{6+(1-\alpha) \omega}\right) \lambda+1=0
$$

where $\omega:=\left(s \ell^{2} / k\right)=\left(\xi_{o} \ell\right)^{2}$ is a dimensionless element number. The solution to Equation (11) can be expressed as follows:

$$
\lambda:=\mathrm{e}^{\mathrm{i} \xi^{h} \ell}=f_{\alpha} \pm \sqrt{\left(f_{\alpha}\right)^{2}-1}=f_{\alpha} \pm \mathrm{i} \sqrt{1-\left(f_{\alpha}\right)^{2}}, \quad f_{\alpha}:=\left(\frac{6-(2+\alpha) \omega}{6+(1-\alpha) \omega}\right)
$$

Note that if $\left|f_{\alpha}\right| \leqslant 1$, then the solution given by Equation (12) is real (i.e. $\xi^{h} \in \mathbb{R}$ ). This solution can be expressed as a series expansion in terms of $\omega$ as follows:

$$
\begin{aligned}
\xi^{h} \ell= & \cos ^{-1}\left(f_{\alpha}\right)=\cos ^{-1}\left(\frac{6-(2+\alpha) \omega}{6+(1-\alpha) \omega}\right) \\
= & \sqrt{\omega}\left[1-\left(\frac{2 \alpha-1}{24}\right) \omega+\left(\frac{20 \alpha^{2}-20 \alpha+9}{1920}\right) \omega^{2}\right. \\
& \left.+\left(\frac{280 \alpha^{3}-420 \alpha^{2}+378 \alpha-103}{193536}\right) \omega^{3}+O\left(\omega^{4}\right)\right]
\end{aligned}
$$

\footnotetext{
${ }^{\S}$ By classical FDM, we refer to the central difference scheme.
} 
If the expression for $\alpha$ is written as a generic series expansion in terms of $\omega$ given by $\alpha=$ $\sum_{m=0}^{\infty} a_{m} \omega^{\mathrm{m}}$, then the solution $\xi^{h}$ can be written as follows:

$$
\begin{aligned}
\xi^{h} \ell= & \sqrt{\omega}\left[1+\left(\frac{1-2 a_{0}}{24}\right) \omega+\left(\frac{20 a_{0}^{2}-20 a_{0}+9}{1920}+\frac{a_{1}}{12}\right) \omega^{2}\right. \\
& \left.+\left(\frac{280 a_{0}^{3}-420 a_{0}^{2}+378 a_{0}-103}{193536}+\frac{\left(2 a_{0}-1\right) a_{1}}{48}+\frac{a_{1}}{12}\right) \omega^{3}+O\left(\omega^{4}\right)\right]
\end{aligned}
$$

where $a_{m}$ are coefficients independent of $\omega$. The relative phase error of the above solution can be expressed as follows:

$$
\frac{\xi^{h}-\xi_{o}}{\xi_{o}}=\frac{\xi^{h} \ell-\sqrt{\omega}}{\sqrt{\omega}}=\left[\left(\frac{1-2 a_{0}}{24}\right) \omega+\left(\frac{20 a_{0}^{2}-20 a_{0}+9}{1920}+\frac{a_{1}}{12}\right) \omega^{2}+O\left(\omega^{3}\right)\right]
$$

Note that for the choice $a_{0}=\frac{1}{2}$, the relative phase error diminishes at the rate of $O\left(\omega^{2}\right)$ or equivalently $O\left(\left(\xi_{o} \ell\right)^{4}\right)$. Further, making the choice $a_{1}=-\frac{1}{40}$, the relative phase error now diminishes at the rate of $O\left(\omega^{3}\right)$ or equivalently $O\left(\left(\xi_{0} \ell\right)^{6}\right)$. Fortunately in $1 \mathrm{D}$, it is possible to choose $\alpha$ such that the solution given by Equation (12) is nodally exact (i.e. $\xi^{h} \ell=\xi_{0} \ell=\sqrt{\omega}$ ). The expression for $\alpha$ that reproduces this effect, say $\alpha_{e}$, can be written as follows:
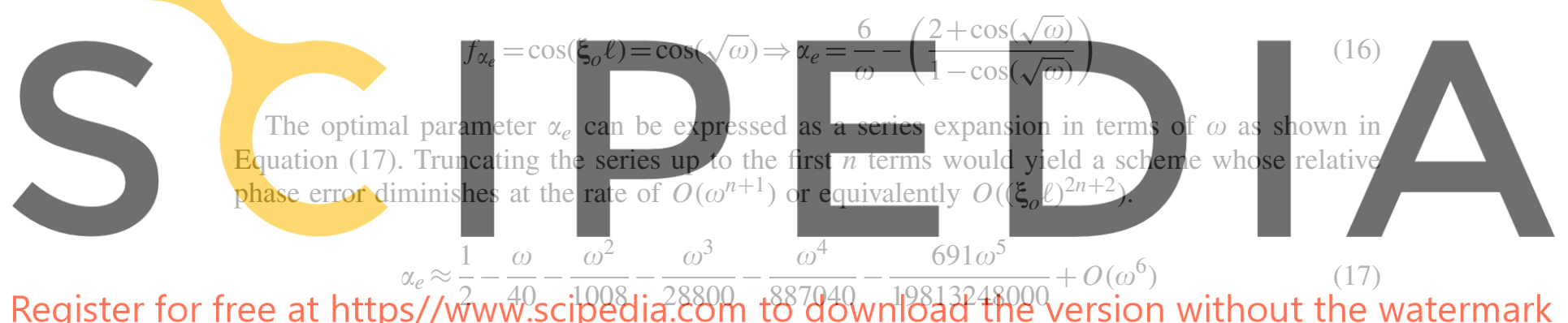

Register for free at https//www.scipedia.com t8 downiload2 the version without the watermark

\subsection{Dispersion plots in ID}

In this section, we consider $\alpha \in\left\{0,1,0.5, \alpha_{e}\right\}$ and study their dispersion plots. The subscripts $c$, $l, m$ are flags used for the expressions obtained using $\alpha=\{0,1,0.5\}$ respectively. These cases correspond for the stencils that arise using the consistent, lumped and mixed (higher-order) mass matrices, respectively. The subscript $e$ is used to flag the choice $\alpha=\alpha_{e}$, the optimal expression for $\alpha$, in order to attain nodally exact numerical solutions in 1D. For the graphical representation of $f(\omega)$ and $\xi(\omega)$, we normalize some of these fields as follows:

$$
\omega^{*}:=\frac{\omega}{\pi^{2}}, \quad \xi^{*}:=\frac{\xi}{\xi_{\mathrm{nq}}}=\frac{\xi \ell}{\pi}
$$

Restricting the domain to $\omega^{*} \in[0,1]$ guarantees that the Nyquist frequency ${ }^{\mathbb{I I}}$ of the discretization $\left(\left(\xi_{\text {nq }}\right)\right)$ is always greater than the frequency of the exact solution $\left(\xi_{o}\right)$. Thus, for every wave length of the harmonic solution we ensure the presence of at least two elements. The Nyquist-Shannon sampling theorem states that this minimum resolution of the mesh is essential to allow a perfect reconstruction of the solution using sinusoidal interpolation. However, using linear interpolation at least four elements per wavelength $\left(\xi_{o} \ell \leqslant(\pi / 2)\right.$ or $\left.\omega^{*} \leqslant\left(\frac{1}{4}\right)\right)$ are needed to capture the sinusoidal profile. As a rule of thumb, at least 8 to 10 elements per wavelength are recommended for a decent

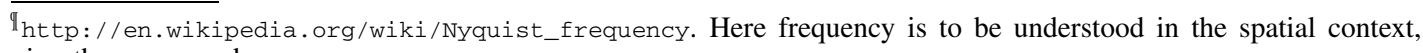
i.e. the wavenumber. 


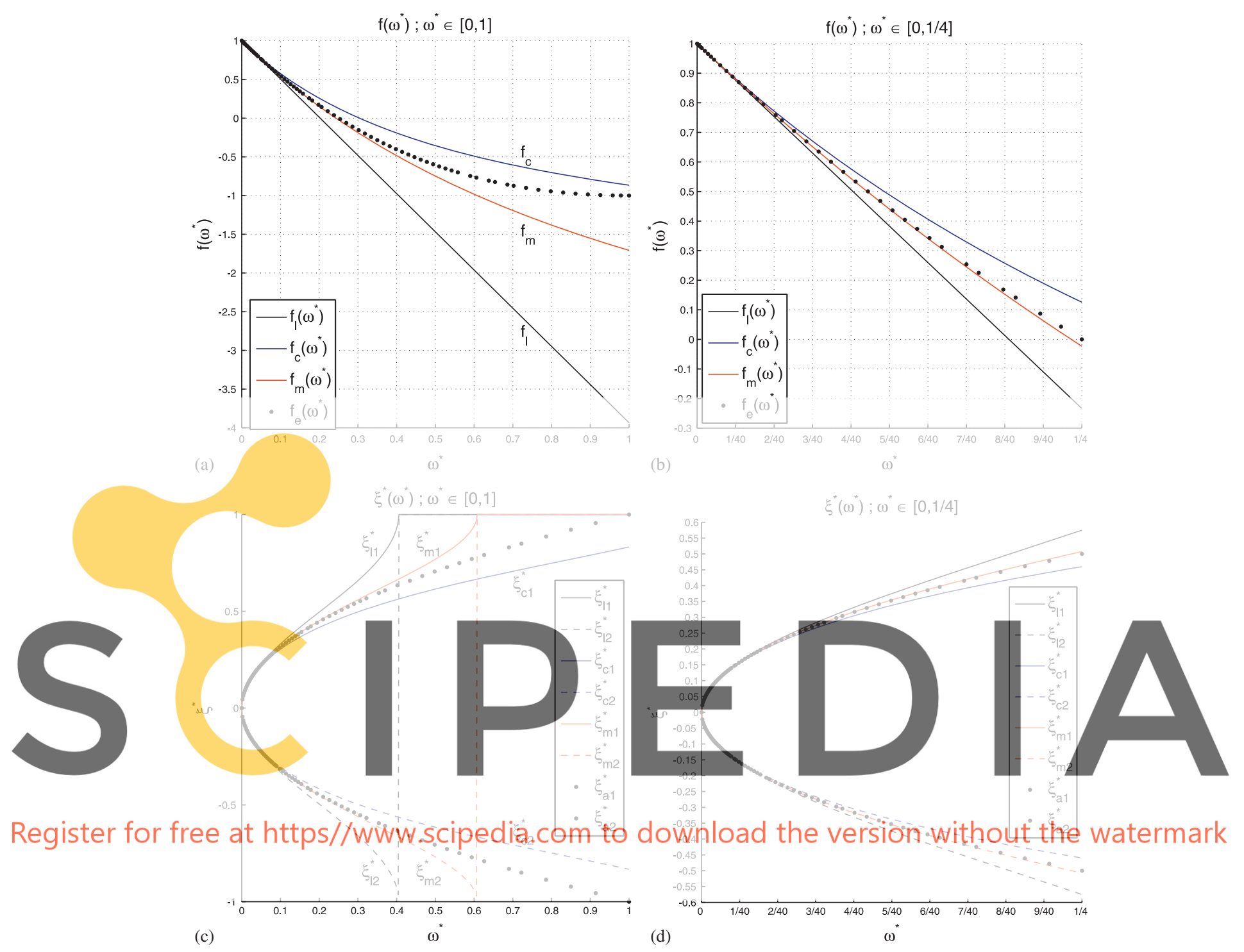

Figure 1. Plots of $f\left(\omega^{*}\right)$ and $\xi^{*}\left(\omega^{*}\right)$ : (a) domain: $\omega^{*} \in[0,1]$; (b) domain: $\omega^{*} \in\left[0, \frac{1}{4}\right]$;

(c) domain: $\omega^{*} \in[0,1]$; and (d) domain: $\omega^{*} \in\left[0, \frac{1}{4}\right]$.

representation of the solution using linear interpolation [11,65]. The latter resolution of the mesh is guaranteed by restricting the domain to $\omega^{*} \in\left[0, \frac{1}{16}\right]$.

Figures 1 (a) and (b) illustrate the plot of $f\left(\omega^{*}\right)$ for $\omega^{*} \in[0,1]$ and $\omega^{*} \in\left[0, \frac{1}{4}\right]$, respectively. As expected a higher-order convergence of $f_{m} \rightarrow f_{e}$ is observed as $\omega^{*} \rightarrow 0$. Also for both the domains $f\left(\omega^{*}\right) \leqslant 0$ and in particular for the latter domain i.e. $\omega^{*} \in\left[0, \frac{1}{4}\right]$, we see that $\left|f\left(\omega^{*}\right)\right|<1$. Figures 1 (c) and (d) illustrate the plot of $\xi^{*}\left(\omega^{*}\right)$ for $\omega^{*} \in[0,1]$ and $\omega^{*} \in\left[0, \frac{1}{4}\right]$, respectively. Whenever $\left|f\left(\omega^{*}\right)\right|>1$, Equation (12) suggests that $\lambda:=\exp \left(i \xi^{h} \ell\right) \in \mathbb{R}$. This implies that $\xi^{h}$ is a complex number $\left(\xi^{h} \in \mathbb{C}\right)$ with the real part $\Re\left(\xi^{h}\right)=(n \pi / \ell), n \in\{0,1,2, \ldots\}$ and the imaginary part $\Im(\xi) \neq 0$. As the Nyquist frequency in space is $\xi_{\text {nq }}=\pi / \ell$, the real part is either $\Re\left(\xi^{h}\right)=0$ for $f\left(\omega^{*}\right) \geqslant 0$ or $\Re\left(\xi^{h}\right)=(\pi / \ell)$ for $f\left(\omega^{*}\right)<0$. Thus, whenever $\left|f\left(\omega^{*}\right)\right|>1$ we find $\Re\left(\xi^{h}\right)=(\pi / \ell)$, i.e. $\Re\left(\xi^{h *}\right)=1$ (see Figure $1(\mathrm{c}))$. Also as $\Im(\xi) \neq 0$, the numerical solutions will be subjected to amplification intrinsic to the discretization (the one studied in the von Neumann analysis). Finally, whenever $\left|f\left(\omega^{*}\right)\right| \leqslant 1$, the solution $\xi^{h}$ is real $\left(\xi^{h} \in \mathbb{R}\right)$ and all the considered schemes are devoid of any amplification 


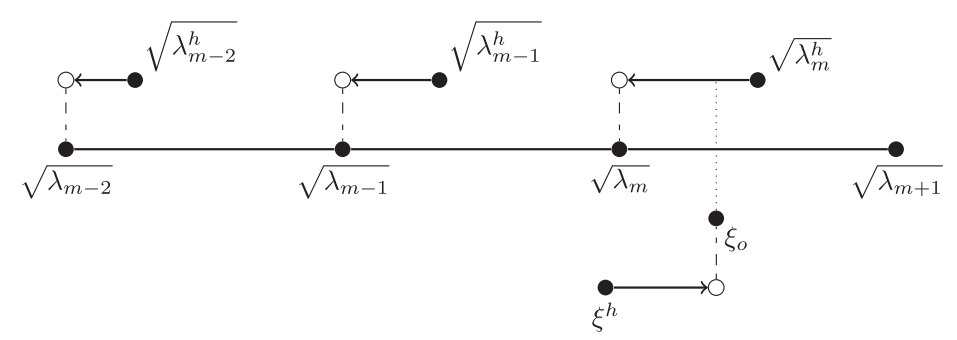

Figure 2. A schematic diagram that illustrates the encounter of a zone of degeneracy on mesh refinement $(\ell \rightarrow 0)$. As the value of $\sqrt{\lambda_{m}^{h}}$ crosses $\xi_{o}$ on its path toward $\sqrt{\lambda_{m}}$, the discrete LBB constant takes values arbitrarily close to zero.

intrinsic to the discretization. In Figure 1(d) we observe that for $\omega^{*} \in\left[0, \frac{1}{16}\right]$, the graphs of $\xi_{e}^{*}$ and $\xi_{m}^{*}$ are indistinguishable.
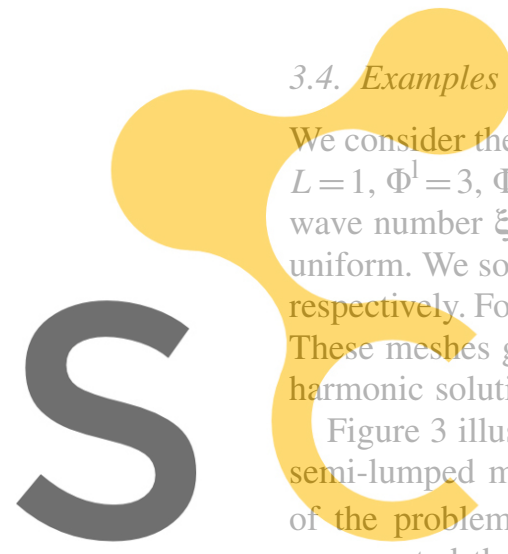

We consider the problem defined in Equation (5) with the following problem data: $k=1 \mathrm{e}-3, s=1$, $L=1, \Phi^{1}=3, \Phi^{\mathrm{r}}=1$. Thus, the exact solution of the problem given by Equation (6) has an angular wave number $\xi_{o}=10 \sqrt{10}$. The discretization of the space is done by linear finite elements and is uniform. We solve the problem using $\alpha \in\{0,1,0.5\}$ and the subscripts $c, l, m$ are used to flag them, respectively. Four meshes of differentresolutions, viz. 41, 81, 162 and 323 eiements, are considered. These meshes guarantee the presence of at least 8,16,32 and 64 elements per wavelength of the harmonic solution, res Figure 3 illustrates semi-lumped mass matrices of the problem denoted by $\Phi^{\mathrm{a}}$. In Figure 3(a), the solutions as expected the phase accuracy improves on mesh refinement (Figures 3(b)-(d)). We observe a remarkable error in the amplitude of these solutions. Note that there is no.intrinsic amplification

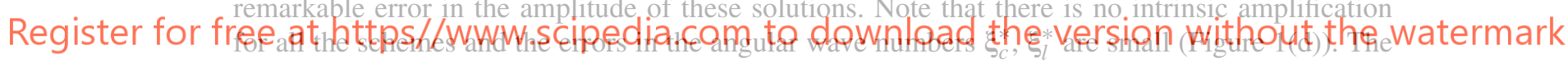
amplitude of the solution depends not only on the intrinsic amplification of the scheme but also on the wave number $\xi$ and on the applied Dirichlet boundary conditions. Thus, we may conclude that small errors in the wave number of the computed solution may result in huge errors in their amplitude. An alternative explanation to this behavior can be given via the following argument. First note that $\left(\sqrt{\lambda_{10}}=31.4159\right)<\left(\xi_{o}=10 \sqrt{10}=31.6227\right)<\left(\sqrt{\lambda_{11}}=34.5575\right)$. It is possible that the discrete eigenvalue $\sqrt{\lambda_{10}^{h}}$ for the initial course mesh/grid is greater than $\xi_{o}$ and on further mesh refinement, it approaches $\sqrt{\lambda_{10}}$ by crossing $\xi_{o}$. This explains the observation that the numerical solution $\Phi_{h}^{\mathrm{c}}$ on mesh refinement first explodes (as it enters the zone of degeneracy) and then gradually converges to the exact solution. Figure 2 illustrates schematicallyll the encounter of a zone of degeneracy on mesh refinement $(\ell \rightarrow 0)$ as the value of $\sqrt{\lambda_{m}^{h}}$ crosses $\xi_{o}$ on its path toward $\sqrt{\lambda_{m}}$. Nevertheless, the convergence of the discrete wavenumber $\left(\xi^{h} \rightarrow \xi_{o}\right)$ need not be affected in this process. This argument also suggests that this phenomenon could have been equally observed for the solutions $\Phi_{h}^{1}$ and $\Phi_{h}^{\mathrm{m}}$ should their corresponding discrete eigenvalues cross $\xi_{o}$.

On the other hand, the solution $\Phi_{h}^{\mathrm{m}}$ could represent approximately the profile of the exact solution even on the coarsest mesh (see Figure 3(a)). Figures 3(b)-(d) show that on further mesh refinements, $\Phi_{h}^{\mathrm{m}}$ is indistinguishable from the analytical solution.

${ }$ A similar figure was presented earlier in [6] (cf. Figure 1, p. 74). 


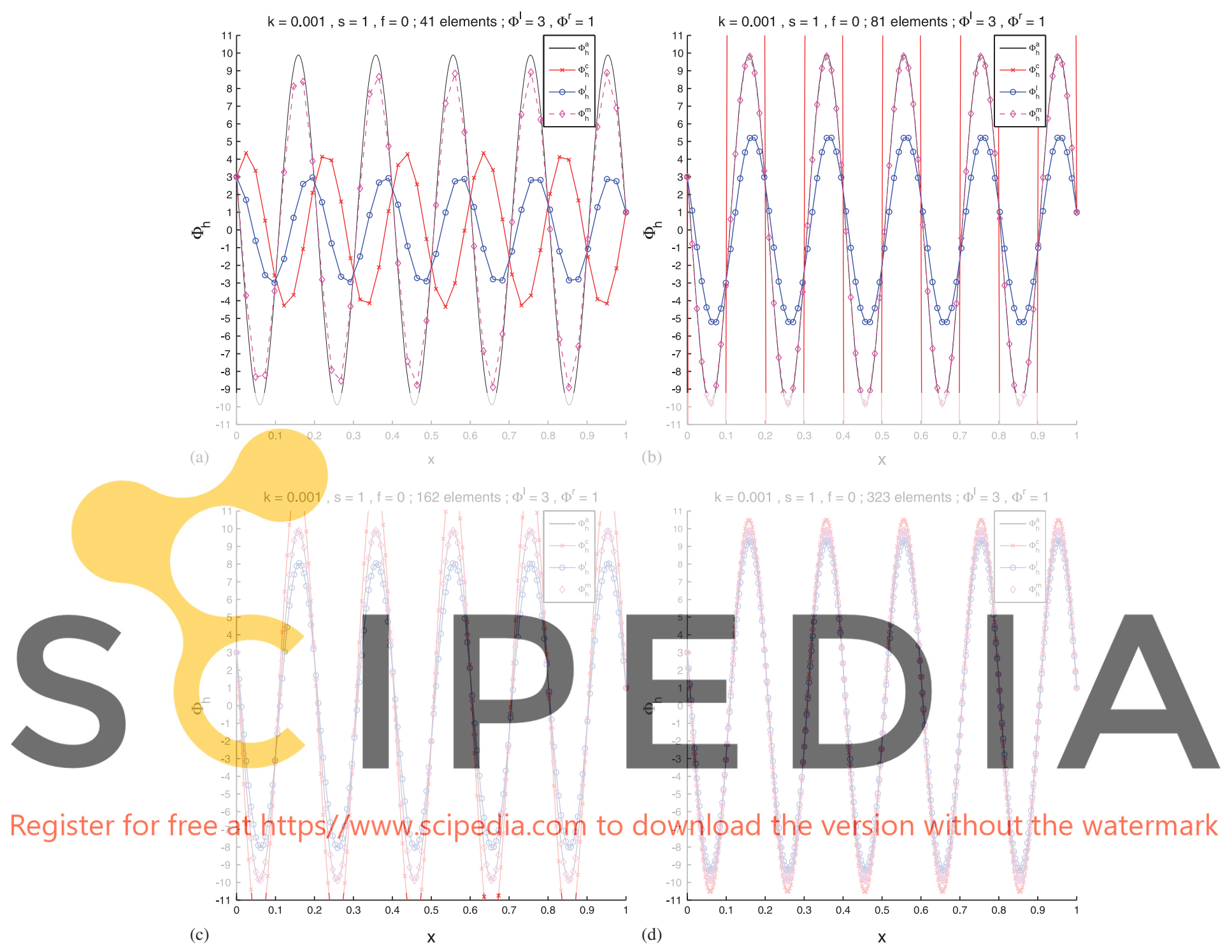

Figure 3. Numerical solution $\Phi_{h}$ using a mesh with at least: (a) 8 elements per wave length; (b) 16 elements per wave length; (c) 32 elements per wave length; and (d) 64 elements per wave length. In Figures (c) and (d), the solution $\Phi_{h}^{\mathrm{m}}$ effectively coincides with the exact solution and the solutions $\Phi_{m}^{\mathrm{c}}$ and $\Phi_{m}^{1}$ bound the exact solution from above and below, respectively.

\section{ANALYSIS IN 2D}

\subsection{Introduction}

In multidimensions, the general solution to the problem (1) considering a linear source $f(\mathbf{x})$ may be expressed as follows:

$$
\begin{aligned}
& \phi(\mathbf{x})=\frac{f}{s}+\sum_{\theta} C_{\theta} \exp \left(i \xi^{\theta} \cdot \mathbf{x}\right) \\
& \left|\xi^{\theta}\right|=\xi_{o} \Rightarrow \xi^{\theta}:=\left(\xi_{1}^{\theta}, \xi_{2}^{\theta}\right)=\left(\xi_{o} \cos (\theta), \xi_{o} \sin (\theta)\right)
\end{aligned}
$$

where $C_{\theta}$ represents a generic constant independent of the spatial coordinates. Generally, it is not possible to arrive at an expression for $C_{\theta}$ in the closed form. Nevertheless, this detail is not 
needed in the Fourier analysis of these problems. Eliminating $\theta$ from Equation (19b) we arrive at the characteristic equation of the continuous problem (1):

$$
\left(\xi_{1}^{\theta}\right)^{2}+\left(\xi_{2}^{\theta}\right)^{2}=\xi_{o}^{2}
$$

\subsection{Galerkin FEM using rectangular bilinear finite elements}

The element contributions to the matrices given in Equation (4) using 4-node rectangular bilinear finite elements are

$$
\begin{aligned}
\mathbf{D}^{e} & =\frac{\ell_{2}}{6 \ell_{1}}\left[\begin{array}{cccc}
2 & -2 & -1 & 1 \\
-2 & 2 & 1 & -1 \\
-1 & 1 & 2 & -2 \\
1 & -1 & -2 & 2
\end{array}\right]+\frac{\ell_{1}}{6 \ell_{2}}\left[\begin{array}{cccc}
2 & 1 & -1 & -2 \\
1 & 2 & -2 & -1 \\
-1 & -2 & 2 & 1 \\
-2 & -1 & 1 & 2
\end{array}\right] \\
\mathbf{M}^{e} & =\frac{\ell_{1} \ell_{2}}{36}\left[\begin{array}{llll}
4 & 2 & 1 & 2 \\
2 & 4 & 2 & 1 \\
1 & 2 & 4 & 2 \\
2 & 1 & 2 & 4
\end{array}\right]
\end{aligned}
$$

where $\ell_{1}, \ell_{2}$ are the corresponding element lengths along the $2 \mathrm{D}$ axes. Restraining the discretization to be uniform, we can arrive at an equation stencil for every interior node of the mesh. We use the following notation to represent a generic compact stencil obtained for the $(i, j)$ node on a rectangular grid:

$$
\left\{\circ^{j+1}, \circ^{j}, \circ^{j-1}\right\} \mathbf{A}\left\{\circ^{i-1}, \circ^{i}, \circ^{i+1}\right\}^{\mathrm{t}}=0
$$

where A represents the matrix of the stencil coefficients. For instance, if the standard mass matrix obtained in the Galerkin FEM is assembled for a structured rectangular mesh, then we may express the stencil as follows:

$$
\begin{aligned}
& \mathbf{A}^{\mathrm{m}}:=\frac{\ell_{1} \ell_{2}}{36}\{1,4,1\}^{\mathrm{t}}\{1,4,1\}=\frac{\ell_{1} \ell_{2}}{36}\left[\begin{array}{ccc}
1 & 4 & 1 \\
4 & 16 & 4 \\
1 & 4 & 1
\end{array}\right]
\end{aligned}
$$

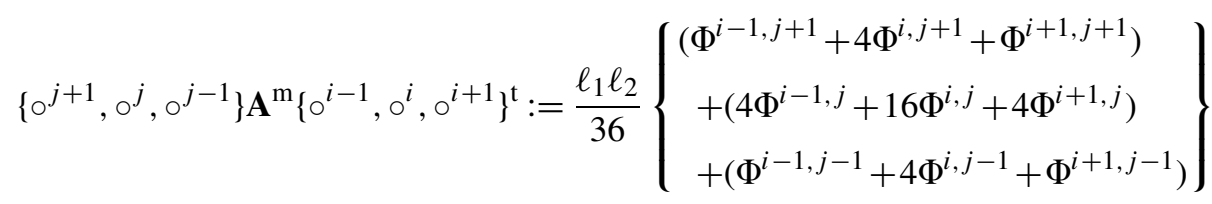

We can guess that a solution to Equation (22) takes the form $\Phi^{i, j}:=\phi\left(x_{1}^{i}, x_{2}^{j}\right)=\exp \left[\mathrm{i}\left(\xi_{1}^{h} x_{1}^{i}+\right.\right.$ $\left.\xi_{2}^{h} x_{2}^{j}\right)$ ]. Substituting this solution into Equation (22) and defining $\lambda_{1}:=\exp \left(i \xi_{1}^{h} \ell_{1}\right)$ and $\lambda_{2}:=$ $\exp \left(i \xi_{2}^{h} \ell_{2}\right)$, we get the characteristic equation of the generic stencil (22):

$$
\left\{\lambda_{2}, 1, \lambda_{2}^{-1}\right\} \mathbf{A}\left\{\lambda_{1}^{-1}, 1, \lambda_{1}\right\}^{\mathrm{t}}=0
$$

The stencil for the Galerkin FEM method corresponding to any interior node $(i, j)$ can be written as Equation (22) with the following definition of the stencil coefficient matrix (A):

$$
\mathbf{A}^{\mathrm{fem}}:=\frac{k \ell_{2}}{6 \ell_{1}}\{1,4,1\}^{\mathrm{t}}\{-1,2,-1\}+\frac{k \ell_{1}}{6 \ell_{2}}\{-1,2,-1\}^{\mathrm{t}}\{1,4,1\}-\frac{s \ell_{1} \ell_{2}}{36}\{1,4,1\}^{\mathrm{t}}\{1,4,1\}
$$


The stencil for the classical FDM method corresponding to any interior node $(i, j)$ can be written as Equation (22) with the following definition of $\mathbf{A}$ :

$$
\mathbf{A}^{\mathrm{fdm}}:=\frac{k \ell_{2}}{6 \ell_{1}}\{0,6,0\}^{\mathrm{t}}\{-1,2,-1\}+\frac{k \ell_{1}}{6 \ell_{2}}\{-1,2,-1\}^{\mathrm{t}}\{0,6,0\}-\frac{s \ell_{1} \ell_{2}}{36}\{0,6,0\}^{\mathrm{t}}\{0,6,0\}
$$

The characteristic equation associated with the stencil for the Galerkin FEM can be written as Equation (25) using the definition of $\mathbf{A}$ given by Equation (26). Likewise, the characteristic equation associated with the stencil for the classical FDM can be written as Equation (25) using the definition of $\mathbf{A}$ given by Equation (27).

\subsection{A nonstandard compact stencil in $2 D$}

Define two free parameters $\alpha_{1}, \alpha_{2}$ and consider the following definition of $\mathbf{A}$ :

$$
\begin{aligned}
\mathbf{A}^{\alpha_{1}, \alpha_{2}}:= & \left(1-\alpha_{1}\right) \frac{k \ell_{2}}{6 \ell_{1}}\{1,4,1\}^{\mathrm{t}}\{-1,2,-1\}+\alpha_{1} \frac{k \ell_{2}}{6 \ell_{1}}\{0,6,0\}^{\mathrm{t}}\{-1,2,-1\} \\
& +\left(1-\alpha_{1}\right) \frac{k \ell_{1}}{6 \ell_{2}}\{-1,2,-1\}^{\mathrm{t}}\{1,4,1\}+\alpha_{1} \frac{k \ell_{1}}{6 \ell_{2}}\{-1,2,-1\}^{\mathrm{t}}\{0,6,0\} \\
& -\left(1-\alpha_{2}\right) \frac{s \ell_{1} \ell_{2}}{36}\{1,4,1\}^{\mathrm{t}}\{1,4,1\}-\alpha_{2} \frac{s \ell_{1} \ell_{2}}{36}\{0,6,0\}^{\mathrm{t}}\{0,6,0\}
\end{aligned}
$$

Note that taking $\alpha_{1}=\alpha_{2}=\alpha$ we arrive at a stencil that is the $\alpha$-interpolation of the FEM and FDM stencils, i.e. $\mathbf{A}^{\alpha, \alpha}=(1-\alpha) \mathbf{A}^{\text {fem }}+\alpha \mathbf{A}^{\text {fdm }}$. Likewise taking $\alpha_{1}=0$ and $\alpha_{2}=\alpha$, we arrive at a stencil that results from the Galerkin FEM method using an $\alpha$-interpolated mass matrix $\mathbf{M}^{\alpha}:=(1-\alpha) \mathbf{M}+\alpha \mathbf{M}_{\mathrm{L}}$. We remark that unlike in 1D where both choices resulted in the same stencil, in 2D the obtained stencils are different.

Next we relate this nonstandard stencil with the compact fourth-order Padé approximation in 2D. A generalized version of the same was studied in [38,39] and the associated stencil coefficient matrix of the scheme $\mathbf{A}^{\gamma}$ can be expressed as follows:

$$
\begin{aligned}
\mathbf{A}^{\gamma}:= & -k\left[\{0,1,0\}+\frac{\{1,-2,1\}}{12}\right]^{\mathrm{t}} \frac{\{1,-2,1\}}{\ell_{1}^{2}}-k \frac{\{1,-2,1\}^{\mathrm{t}}}{\ell_{2}^{2}}\left[\{0,1,0\}+\frac{\{1,-2,1\}}{12}\right] \\
& -s\left[\{0,1,0\}+\frac{\{1,-2,1\}}{12}\right]^{\mathrm{t}}\left[\{0,1,0\}+\frac{\{1,-2,1\}}{12}\right]-s(\gamma-1) \frac{\{1,-2,1\}^{\mathrm{t}}}{12} \frac{\{1,-2,1\}}{12}
\end{aligned}
$$

where $\gamma$ is a free parameter. The standard compact fourth-order Padé scheme in 2D is obtained by selecting $\gamma=1$. Other alternatives viz. $\gamma=0$ and $\gamma=2$ were presented in [66, cf. Appendix, Table VI, p. 542]. After some algebraic rearrangement, matrix $\mathbf{A}^{\gamma}$ given in Equation (29) can be re-written equivalently as follows:

$$
\begin{aligned}
\mathbf{A}^{\gamma}:= & \frac{k}{2}\left[\frac{\{1,4,1\}}{6}+\frac{\{0,6,0\}}{6}\right]^{\mathrm{t}} \frac{\{-1,2,-1\}}{\ell_{1}^{2}}+\frac{k}{2} \frac{\{-1,2,-1\}^{\mathrm{t}}}{\ell_{2}^{2}}\left[\frac{\{1,4,1\}}{6}+\frac{\{0,6,0\}}{6}\right] \\
& -\frac{s}{2}\left[\frac{\{1,4,1\}^{\mathrm{t}}}{6} \frac{\{1,4,1\}}{6}+\frac{\{0,6,0\}^{\mathrm{t}}}{6} \frac{\{0,6,0\}}{6}\right]-s(\gamma-2) \frac{\{1,-2,1\}^{\mathrm{t}}}{12} \frac{\{1,-2,1\}}{12}
\end{aligned}
$$

Note that by selecting $\gamma=2$, we obtain a stencil that is equivalent to the one obtained by taking the average of the FEM and the FDM stencils. Thus,

$$
\mathbf{A}^{2}=\frac{1}{\ell_{1} \ell_{2}} \mathbf{A}^{0.5,0.5}
$$

We now relate this nonstandard stencil for square meshes with the compact scheme proposed by Vichnevetsky and Bowles [67] in order to reduce the anisotropy related to the numerical 
dispersion. This scheme was studied in [65] and the conditions for appropriate numerical isotropy were determined therein. Also, this scheme was used to synthesize an equivalent transmission-line matrix (TLM) [68] model for Maxwell's equations in [69]. The associated stencil coefficient matrix $\mathbf{A}^{v b}$ can be written as follows:

$$
\mathbf{A}^{v b}:=\frac{\gamma k}{\ell^{2}}\left[\begin{array}{ccc}
0 & -1 & 0 \\
-1 & 4 & -1 \\
0 & -1 & 0
\end{array}\right]+\frac{(1-\gamma) k}{2 \ell^{2}}\left[\begin{array}{ccc}
-1 & 0 & -1 \\
0 & 4 & 0 \\
-1 & 0 & -1
\end{array}\right]-s\left[\begin{array}{ccc}
0 & 0 & 0 \\
0 & 1 & 0 \\
0 & 0 & 0
\end{array}\right]
$$

where $\gamma$ is the associated interpolation parameter. Note that for $\gamma=1$, we recover the classical FDM (i.e. the second-order central difference scheme) and for $\gamma=0$, we get a similar scheme but with the stencil inclined at $45^{\circ}$ and hence with the mesh size $\sqrt{2} \ell$. Note that we recover the Galerkin FEM contribution of the term $-k \Delta \phi$ by choosing $\gamma=\left(\frac{1}{3}\right)$. Making the substitution $\gamma=(1+2 \alpha) / 3$ in Equation (32) and after some algebraic rearrangement, matrix $\mathbf{A}^{v b}$ can be re-written equivalently as follows:

$$
\begin{aligned}
\mathbf{A}^{v b}:= & k\left[\frac{(1-\alpha)}{6}\{1,4,1\}+\frac{\alpha}{6}\{0,6,0\}\right]^{\mathrm{t}} \frac{\{-1,2,-1\}}{\ell^{2}} \\
& +k \frac{\{-1,2,-1\}^{\mathrm{t}}}{\ell^{2}}\left[\frac{(1-\alpha)}{6}\{1,4,1\}+\frac{\alpha}{6}\{0,6,0\}\right]-s \frac{\{0,6,0\}^{\mathrm{t}}}{6} \frac{\{0,6,0\}}{6}
\end{aligned}
$$

This is precisely what we get using an $\alpha$-interpolated (Galerkin FEM and classical FDM) diffusion matrix in the classical FDM stencil. Thus, on square meshes we can relate $\mathbf{A}^{v b}$ with the nonstandard stencil as shown as follows:

$$
\mathbf{A}^{v b}=\frac{1}{\ell^{2}} \mathbf{A}^{\alpha, 1}
$$

Using the definition of $\mathbf{A}$ given by Equation (28), the characteristic equation associated with the resulting stencil is given by Equation (35) and on simplification we arrive at Equation (36).

$$
\begin{gathered}
\left\{\lambda_{2}, 1, \lambda_{2}^{-1}\right\} \mathbf{A}^{\alpha_{1}, \alpha_{2}}\left\{\lambda_{1}^{-1}, 1, \lambda_{1}\right\}^{\mathrm{t}}=0 \\
\Rightarrow\left(\frac{\left[\left(1-\alpha_{1}\right)\left(\lambda_{2}^{2}+4 \lambda_{2}+1\right)+6 \alpha_{1} \lambda_{2}\right]\left(-1+2 \lambda_{1}-\lambda_{1}^{2}\right)}{6 \omega_{1}}\right) \\
+\left(\frac{\left(-\lambda_{2}^{2}+2 \lambda_{2}-1\right)\left[\left(1-\alpha_{1}\right)\left(1+4 \lambda_{1}+\lambda_{1}^{2}\right)+6 \alpha_{1} \lambda_{1}\right]}{6 \omega_{2}}\right) \\
-\left(\frac{\left[\left(1-\alpha_{2}\right)\left(\lambda_{2}^{2}+4 \lambda_{2}+1\right)\left(1+4 \lambda_{1}+\lambda_{1}^{2}\right)+36 \alpha_{2} \lambda_{2} \lambda_{1}\right]}{36}\right)=0
\end{gathered}
$$

where $\omega_{1}, \omega_{2}$ are two dimensionless element numbers defined as follows:

$$
\omega_{1}:=\frac{s \ell_{1}^{2}}{k}=\left(\xi_{o} \ell_{1}\right)^{2}, \quad \omega_{2}:=\frac{s \ell_{2}^{2}}{k}=\left(\xi_{o} \ell_{2}\right)^{2}
$$

Unlike in 1D, the characteristic equations of the stencils in 2D have infinite solutions (fundamental frequencies $\left.\left(\xi_{1}^{h}, \xi_{2}^{h}\right)\right)$ for every $\left(\omega_{1}, \omega_{2}\right)$ pair. For every choice of the pair $\left(\omega_{1}, \omega_{2}\right)$, these solutions will trace well-defined contours in the $\xi_{1}^{h}-\xi_{2}^{h}$ plane. The solutions to Equation (36) are symmetric about the origin and the axes. This statement can be easily verified due to the fact that by replacing the pair $\left(\lambda_{1}, \lambda_{2}\right)$ with $\left(\lambda_{1}^{ \pm 1}, \lambda_{2}^{ \pm 1}\right)$ in Equation (36), we end up in the same 
equation. Thus, we may conclude that if $\left(\xi_{1}^{h}, \xi_{2}^{h}\right)$ is a solution to Equation (36), then $\left( \pm \xi_{1}^{h}, \pm \xi_{2}^{h}\right)$ are also solutions to the same. Obviously, this statement also extends to the characteristic equation of the continuous problem (20), which additionally has a rotational symmetry (i.e. if $\left(\xi_{1}^{\theta}, \xi_{2}^{\theta}\right)$ is a solution, then $\left(\xi_{2}^{\theta}, \xi_{1}^{\theta}\right)$ is also a solution). These contour lines are circular for the continuous problem and their radius equals to the chosen $\xi_{o}$ value. Rotational symmetry for the solution $\left(\xi_{1}^{h}, \xi_{2}^{h}\right)$ is attained if the element lengths are the same, i.e. $\ell_{1}=\ell_{2}=\ell$. In this case, the stencil coefficient matrix $\mathbf{A}^{\alpha_{1}, \alpha_{2}}$ is symmetric and after scaling down by $k$ it can be expressed as follows:

$$
\frac{\mathbf{A}^{\alpha_{1}, \alpha_{2}}}{k}=\left[\begin{array}{lll}
A_{2} & A_{1} & A_{2} \\
A_{1} & A_{0} & A_{1} \\
A_{2} & A_{1} & A_{2}
\end{array}\right]^{\alpha_{1}, \alpha_{2}} \quad \begin{aligned}
A_{0}^{\alpha_{1}, \alpha_{2}} & :=\frac{8}{3}-\frac{4 \omega}{9}+\frac{4 \alpha_{1}}{3}-\frac{5 \omega \alpha_{2}}{9} \\
A_{1}^{\alpha_{1}, \alpha_{2}} & :=-\frac{1}{3}-\frac{\omega}{9}-\frac{2 \alpha_{1}}{3}+\frac{\omega \alpha_{2}}{9} \\
A_{2}^{\alpha_{1}, \alpha_{2}} & :=-\frac{1}{3}-\frac{\omega}{36}+\frac{\alpha_{1}}{3}+\frac{\omega \alpha_{2}}{36}
\end{aligned}
$$

where $\omega:=\left(s \ell^{2} / k\right)=\left(\xi_{o} \ell\right)^{2}$.

Finally, we relate this nonstandard stencil with methods that have a symmetric stencil coefficient matrix $\mathbf{A}^{\mathrm{sym}}$ defined as:

$$
\mathbf{A}^{\mathrm{sym}}:=\left[\begin{array}{ccc}
A_{2} & A_{1} & A_{2} \\
A_{1} & A_{0} & A_{1} \\
A_{2} & A_{1} & A_{2}
\end{array}\right]
$$

Let $g_{1}=\left(4 A_{1} / A_{0}\right)$ and $g_{2}=\left(4 A_{2} / A_{0}\right)$ and if $\left(g_{1}+g_{2}+1\right) \neq 0$, then we can obtain $\mathbf{A}^{\text {sym }}$ (possibly scaled by a factor) from $\mathbf{A}^{\alpha_{1}, \alpha_{2}}$ by selecting $\alpha_{1}$ and $\alpha_{2}$ as follows:

$$
\alpha_{1}:=\frac{4\left(g_{1}+g_{2}+1\right)+\omega\left(g_{1}-4 g_{2}\right)}{8\left(g_{1}+g_{2}+1\right)}, \quad \alpha_{2}:=\frac{12\left(g_{1}+g_{2}+1\right)+\omega\left(2-g_{1}-4 g_{2}\right)}{2 \omega\left(g_{1}+g_{2}+1\right)}
$$

For instance, consider the QSFEM method [15] for which the expressions for $g_{1}$ and $g_{2}$ can be written as follows:

$$
\begin{aligned}
g_{1}:=\frac{2\left(c_{1} s_{1}-c_{2} s_{2}\right)}{c_{2} s_{2}\left(c_{1}+s_{1}\right)-c_{1} s_{1}\left(c_{2}+s_{2}\right)}, & g_{2}:=\frac{\left(c_{2}+s_{2}-c_{1}-s_{1}\right)}{c_{2} s_{2}\left(c_{1}+s_{1}\right)-c_{1} s_{1}\left(c_{2}+s_{2}\right)} \\
c_{1}:=\cos \left[\sqrt{\omega} \cos \left(\frac{\pi}{16}\right)\right], & c_{2}:=\cos \left[\sqrt{\omega} \cos \left(\frac{3 \pi}{16}\right)\right] \\
s_{1}:=\cos \left[\sqrt{\omega} \sin \left(\frac{\pi}{16}\right)\right], & s_{2}:=\cos \left[\sqrt{\omega} \sin \left(\frac{3 \pi}{16}\right)\right]
\end{aligned}
$$

\subsection{Numerical solution, phase error and local truncation error}

In this section, we will deal only with the case when $\ell_{1}=\ell_{2}=\ell$. Here we present the solution to Equation (36) for a given $\alpha_{1}, \alpha_{2}$ expressed as a generic series expansion in terms of $\omega$ as follows:

$$
\begin{aligned}
& \alpha_{1}:=\sum_{m=0}^{\infty} a_{m} \omega^{\mathrm{m}} \approx a_{0}+a_{1} \omega+a_{2} \omega^{2}+a_{3} \omega^{3}+O\left(\omega^{4}\right) \\
& \alpha_{2}:=\sum_{m=0}^{\infty} b_{m} \omega^{\mathrm{m}} \approx b_{0}+b_{1} \omega+b_{2} \omega^{2}+b_{3} \omega^{3}+O\left(\omega^{4}\right)
\end{aligned}
$$




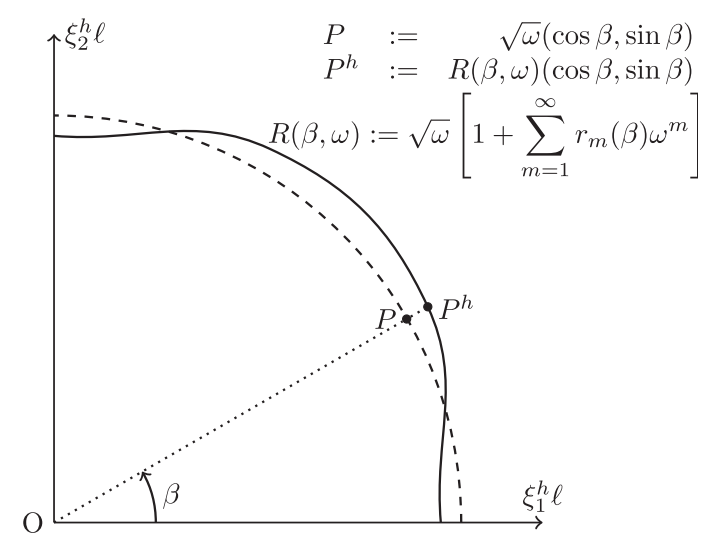

Figure 4. A schematic diagram of the contours traced by the numerical solution $P^{h}\left(\xi_{1}^{h} \ell, \xi_{2}^{h} \ell\right)$ and the exact solution $P\left(\xi_{1}^{\beta} \ell, \xi_{2}^{\beta} \ell\right)$.

where $a_{m}, b_{m}$ are coefficients independent of $\omega$. Following [10,15], the solution $\xi^{h}:=\left(\xi_{1}^{h}, \xi_{2}^{h}\right)$ can also be expressed as a series expansion in terms of $\omega$ :

$$
\begin{aligned}
\left\{\begin{array}{l}
\xi_{1}^{h} \ell \\
\xi_{2}^{h} \ell
\end{array}\right\} & =R\left(a_{m}, b_{m}, \beta, \omega\right)\left\{\begin{array}{c}
\cos (\beta) \\
\sin (\beta)
\end{array}\right\} \\
R & :=\sqrt{\omega}\left[1+\sum_{m=1}^{\infty} r_{m}\left(a_{i}, b_{i}, \beta\right) \omega^{\mathrm{m}}\right] \approx \sqrt{\omega}\left[1+r_{1} \omega+r_{2} \omega^{2}+r_{3} \omega^{3}+O\left(\omega^{4}\right)\right]
\end{aligned}
$$

where $r_{m}$ are coefficients independent of $\omega$ and will be determined later in this section. Recall that the numerical solution in 1D given by Equations (13) or (14) obeys the above series expansion in terms of $\omega$. Figure 4 illustrates schematically the contour traced by the numerical solution $P^{h}\left(\xi_{1}^{h} \ell, \xi_{2}^{h} \ell\right)$ and compares it with the contour of the exact solution $P\left(\xi_{1}^{\beta} \ell, \xi_{2}^{\beta} \ell\right)$. In $[10,15]$ the denomination ' $\operatorname{dist}(\beta)$ ' was used for the distance between $P^{h}$ and $P$, i.e. $\operatorname{dist}(\beta):=R-\sqrt{\omega}$. Therein ' $\operatorname{dist}(\beta)$ ' was used as a measure of the approximation quality of the solution and from it error estimates were derived that bound the solution from below. The relative phase error of the solution along any direction $\beta$ is given by

$$
\frac{\left\|\xi^{h}\right\|-\left\|\xi^{\beta}\right\|}{\left\|\xi^{\beta}\right\|}=\frac{R-\sqrt{\omega}}{\sqrt{\omega}}=\frac{\operatorname{dist}(\beta)}{\sqrt{\omega}}=\sum_{m=1}^{\infty} r_{m} \omega^{\mathrm{m}} \approx\left[r_{1} \omega+r_{2} \omega^{2}+r_{3} \omega^{3}+O\left(\omega^{4}\right)\right]
$$

Substituting $P^{h}\left(\xi_{1}^{h} \ell, \xi_{2}^{h} \ell\right)$ into the stencil corresponding to $\mathbf{A}^{\alpha_{1}, \alpha_{2}}$ given in Equation (38), we get:

$$
A_{0}^{\alpha_{1}, \alpha_{2}}+2 A_{1}^{\alpha_{1}, \alpha_{2}}[\cos (R \cos \beta)+\cos (R \sin \beta)]+4 A_{2}^{\alpha_{1}, \alpha_{2}} \cos (R \cos \beta) \cos (R \sin \beta)=0
$$

Using the definitions of $\alpha_{1}, \alpha_{2}$ and $R$ given in Equations (42) and (43b), respectively, the left-hand side (LHS) of Equation (45) can be expanded as a series in terms of $\omega$ as shown in Equation (46a). The first four coefficients of this series can be expressed as shown in Equations (46b) and (46c), respectively.

$$
\begin{aligned}
\mathrm{LHS} & =\sum_{m=0}^{\infty} S_{m}\left(a_{i}, b_{j}, r_{k}, \beta\right) \omega^{\mathrm{m}} \\
S_{0} & =S_{1}=0, \quad S_{2}=2 r_{1}+\left(\frac{3+2 a_{0}-8 b_{0}}{48}\right)+\left(\frac{1-2 a_{0}}{48}\right) \cos (4 \beta)
\end{aligned}
$$




$$
\begin{aligned}
S_{3}= & 2 r_{2}+r_{1}^{2}+\left(\frac{2 a_{0}-4 b_{0}-1}{12}\right) r_{1}+\left(\frac{24 a_{1}-96 b_{1}-2 a_{0}+8 b_{0}-5}{576}\right) \\
& +\left[\left(\frac{10 a_{0}-7-120 a_{1}}{2880}\right)+\left(\frac{1-2 a_{0}}{12}\right) r_{1}\right] \cos (4 \beta)
\end{aligned}
$$

The local truncation error of the solution along any direction $\beta$ is found by substituting the exact solution $P\left(\xi_{1}^{\beta} \ell, \xi_{2}^{\beta} \ell\right)$ into the stencil corresponding to $\mathbf{A}^{\alpha_{1}, \alpha_{2}}$ given in Equation (38). This is equivalent to substituting $r_{k}=0 \forall k$ in the expression for LHS given in Equation (46a). Thus using the result $S_{0}=S_{1}=0$, the relative truncation error $\mathbb{T}$ along any direction $\beta$ is given by:

$$
\mathbb{T}:=\frac{\left.\mathrm{LHS}\right|_{P}}{\omega}=\sum_{2}^{\infty} S_{m}\left(a_{i}, b_{j}, r_{k}=0, \beta\right) \omega^{m-1} \approx\left[S_{2} \omega+S_{3} \omega^{2}+S_{4} \omega^{3}+O\left(\omega^{4}\right)\right]
$$

We now present the expressions for the unknowns $r_{k}$. Clearly all the coefficients $S_{m}$ should be zero for Equation (45) to hold. We can solve the unknowns $r_{k}$ by imposing the conditions $S_{m}=0 \forall m$. Thus, the first two unknowns in Equation (43b) viz. $r_{1}$ and $r_{2}$ can be expressed as follows:

$$
\begin{aligned}
r_{1}= & -\left(\frac{3+2 a_{0}-8 b_{0}}{96}\right)-\left(\frac{1-2 a_{0}}{96}\right) \cos (4 \beta) \\
r_{2}= & -\frac{r_{1}^{2}}{2}-\left(\frac{2 a_{0}-4 b_{0}-1}{24}\right) r_{1}-\left(\frac{24 a_{1}-96 b_{1}-2 a_{0}+8 b_{0}-5}{1152}\right) \\
& -\left[\left(\frac{10 a_{0}-7-120 a_{1}}{5760}\right)+\left(\frac{1-2 a_{0}}{24}\right) r_{1}\right] \cos (4 \beta)
\end{aligned}
$$

Note that we obtain the condition $r_{1}=0$ if and only if $a_{0}$ and $b_{0}$ satisfy the condition $a_{0}=b_{0}=\left(\frac{1}{2}\right)$. Further, we obtain the condition $r_{2}=0$ if and only if $a_{1}$ and $b_{1}$ satisfy the condition $a_{1}=\left(-\frac{1}{60}\right)$ and $b_{1}=\left(-\frac{1}{40}\right)$. For these choices of $a_{0}, a_{1}, b_{0}$ and $b_{1}$, the first five coefficients in $\left\{S_{m}\right\}$ can be simplified as follows:

$$
\begin{aligned}
S_{0}= & S_{1}=0, \quad S_{2}=2 r_{1}, \quad S_{3}=2 r_{2}+r_{1}^{2}-\frac{r_{1}}{6} \\
S_{4}= & 2 r_{3}+2 r_{1} r_{2}-\frac{r_{2}}{6}-\frac{r_{1}}{720}-\frac{5 r_{1}^{2}}{12} \\
& -\left[\frac{5}{55296}-\left(\frac{a_{2}-4 b_{2}}{24}\right)+\left(\frac{1+576 a_{2}}{13824}\right) \cos (4 \beta)+\frac{\cos (8 \beta)}{387072}\right]
\end{aligned}
$$

Likewise, by imposing the condition $S_{4}=0$ the unknown $r_{3}$ in Equation (43b) can be simplified to the following:

$$
r_{3}=\left[\frac{5}{110592}-\left(\frac{a_{2}-4 b_{2}}{48}\right)+\left(\frac{1+576 a_{2}}{27648}\right) \cos (4 \beta)+\frac{\cos (8 \beta)}{774144}\right]
$$

Clearly, it is impossible to obtain the condition $r_{3}=0$ and this fact was pointed out earlier in $[15,10]$. To conclude this section, we summarize the salient results. The parameters $\alpha_{1}$ and $\alpha_{2}$ that appear in $\mathbf{A}^{\alpha_{1}, \alpha_{2}}$ can be chosen such that the numerical solution is sixth-order accurate, i.e. $O\left(\left(\xi_{o} \ell\right)^{6}\right)$ or equivalently $O\left(\omega^{3}\right)$. Recall that this is the maximum order of accuracy that can be attained on any compact stencil $[10,15]$. All such $\alpha_{1}$ and $\alpha_{2}$ should obey the following series expansion in terms of $\omega$ :

$$
\alpha_{1}=\frac{1}{2}-\frac{\omega}{60}+\sum_{m=2}^{\infty} a_{m} \omega^{\mathrm{m}}, \quad \alpha_{2}=\frac{1}{2}-\frac{\omega}{40}+\sum_{m=2}^{\infty} b_{m} \omega^{\mathrm{m}}
$$


The relative phase and local truncation errors of these schemes can be expressed as follows:

$$
\frac{\left\|\xi^{h}\right\|-\left\|\xi^{\beta}\right\|}{\left\|\xi^{\beta}\right\|}=r_{3} \omega^{3}+O\left(\omega^{4}\right), \quad \mathbb{T}=-2 r_{3} \omega^{3}+O\left(\omega^{4}\right)
$$

where $r_{3}$ is given in Equation (50). As $a_{m}, b_{m}(m \geqslant 2)$ can be chosen arbitrarily, infinitely many sixth-order schemes can be designed through $\mathbf{A}^{\alpha_{1}, \alpha_{2}}$. Of course, some particular choice of $a_{m}, b_{m}$ may yield a scheme with better features. For instance, $a_{m}, b_{m}$ may be chosen such that the local truncation error $\mathbb{T}$ is zero along some chosen directions.

\section{5. $\alpha$-interpolation of the FEM and the FDM in $2 D$}

In this section we consider the case $\alpha_{1}=\alpha_{2}=\alpha$, which results in a scheme that is the $\alpha$-interpolation of the FEM and the FDM stencils. Here the coefficients $a_{m}=b_{m} \forall m$. Recall that a necessary condition to obtain a sixth-order scheme is $a_{1}=\left(-\frac{1}{60}\right)$ and $b_{1}=\left(-\frac{1}{40}\right)$. Thus, an immediate consequence is that this $\alpha$-interpolation scheme can be at the best fourth-order accurate. Nevertheless, a compromise to the loss in accuracy is that the condition $\alpha_{1}=\alpha_{2}=\alpha$ imposes an additional structure to the scheme that may be exploited. For instance, this additional structure might throw light on the extension of this scheme to unstructured meshes. Precisely, if it is possible to design a PetrovGalerkin method that would yield the FDM stencil on a structured mesh, then this scheme can be extended to unstructured meshes in a straightforward manner. We show that indeed it is possible to design such a Petrov-Galerkin method using just the lowest-order block finite elements [70].

We now discuss the salient features of this scheme, i.e. the case $\alpha_{1}=\alpha_{2}=\alpha$. It is possible to choose $\alpha$ such that the local truncation error along any direction $\theta$ is zero. Let this choice be denominated as $\alpha_{\theta}$ and it can be expressed as follows:

$$
\alpha_{\theta}:=\frac{6\left(c_{\theta}+s_{\theta}+2 c_{\theta} s_{\theta}-4\right)+\omega\left(2 c_{\theta}+2 s_{\theta}+c_{\theta} s_{\theta}+4\right)}{12\left(1-c_{\theta}-s_{\theta}+c_{\theta} s_{\theta}\right)+\omega\left(2 c_{\theta}+2 s_{\theta}+c_{\theta} s_{\theta}-5\right)}, \quad \begin{array}{cl}
c_{\theta}:=\cos (\sqrt{\omega} \cos (\theta)) \\
s_{\theta}:=\cos (\sqrt{\omega} \sin (\theta))
\end{array}
$$

Note that choosing $\theta=0$ we would recover the expression for $\alpha$ given in Equation (16), which results in solutions that are nodally exact in 1D. The expression for $\alpha_{\theta}$ can be written as a series expansion in terms of $\omega$ as shown as follows:

$$
\alpha_{\theta}=\sum_{m=0}^{\infty} a_{m} \omega^{\mathrm{m}} \approx \frac{1}{2}-\left[\frac{5+\cos (4 \theta)}{3+\cos (4 \theta)}\right] \frac{\omega}{60}-\left[\frac{35+28 \cos (4 \theta)+\cos (8 \theta)}{3+\cos (4 \theta)}\right] \frac{\omega^{2}}{16128}+O\left(\omega^{3}\right)
$$

Recall that the choice $a_{0}=\left(\frac{1}{2}\right)$ will make the coefficient $r_{1}=0$ and hence using the expression for $\alpha_{\theta}$, we will always obtain fourth-order accurate solutions on uniform meshes. The expression for the coefficient $r_{2}$ given in Equation (48b) can now be simplified to the following.

$$
r_{2}=\frac{1}{1440}\left[\frac{\cos (4 \theta)-\cos (4 \beta)}{3+\cos (4 \theta)}\right]
$$

The relative phase and local truncation errors of this scheme can be expressed as follows:

$$
\frac{\left\|\xi^{h}\right\|-\left\|\xi^{\beta}\right\|}{\left\|\xi^{\beta}\right\|}=r_{2} \omega^{2}+O\left(\omega^{3}\right), \quad \mathbb{T}=-2 r_{2} \omega^{2}+O\left(\omega^{3}\right)
$$

where $r_{2}$ is given in Equation (55). So far the direction $\theta$, along which the local truncation error is made zero, is arbitrary. We now try to optimize the solution error with respect to $\theta$. Ideally, the function to optimize could be either the relative phase or local truncation errors and the optimization problem can be posed as follows:

$$
\min _{\theta} \max _{\beta}|\mathbb{T}| \quad \text { (or) } \min _{\theta} \max _{\beta}\left|\frac{\left\|\xi^{h}\right\|-\left\|\xi^{\beta}\right\|}{\left\|\xi^{\beta}\right\|}\right|
$$


Unfortunately, this is a difficult problem to solve in the closed form as it is a nonlinear function of $\omega$ and the location of the minimum might vary with $\omega$. We conjuncture that in the pre-asymptotic range (i.e. $\xi_{o} \ell \ll 1$ or equivalently $\omega \ll 1$ ), the location of the minimum in the $\theta-\beta$ space is independent of $\omega$. Thus, under this assumption the minimization of the relative phase or local truncation errors is essentially equivalent to the minimization of the coefficient of the lowest-order term, i.e. here $r_{2}$. Hence we choose to optimize the coefficient $r_{2}$ instead. The redefined problem and its solution is given as follows:

$$
\begin{gathered}
\min _{\theta} \max _{\beta}\left|r_{2}\right|=\min _{\theta} \max _{\beta} \frac{|\cos (4 \theta)-\cos (4 \beta)|}{1440(3+\cos (4 \theta))}=\min _{\theta} \frac{1+|\cos (4 \theta)|}{1440(3+\cos (4 \theta))}=\frac{1}{4320} \\
\max _{\beta} \text { occurs at }|\cos (4 \beta)|=1 \Rightarrow \beta=\frac{m \pi}{4}, \quad m=\{0,1,2, \ldots\} \\
\min _{\theta} \text { occurs at }|\cos (4 \theta)|=0 \Rightarrow \theta=\frac{(2 n+1) \pi}{8}, \quad n=\{0,1,2, \ldots\}
\end{gathered}
$$

Thus, for a given $\theta$ the maximum error in the stencil will be found for some $\beta \in\{0,(\pi / 4),(\pi / 2)\}$. That maximum error along the direction $\beta$ takes a minimum value if the chosen direction (where the truncation error is made zero) is some $\theta \in\{(\pi / 8,3 \pi / 8)\}$. Note that due to the inherent symmetries in the stencil, the expressions for $\alpha_{(\pi / 8)}$ and $\alpha_{(3 \pi / 8)}$ are equivalent.

\subsection{Dispersion plots in $2 D$}

For a feasible graphical representation and comparison of the solutions to the characteristic equations, we plot the $\xi_{1}-\xi_{2}$ contours for some values of $\left(\omega_{1}, \omega_{2}\right)$ only. Here and henceforth the superscripts $\{\theta, h\}$ are dropped in order to refer to the contour plots of both the continuous and discrete problems simultaneously. In order to retain generality to the plots, the quantities $\omega_{1}, \omega_{2}$, $\xi_{1}^{\theta}, \xi_{2}^{\theta}, \xi_{1}^{h}$ and $\xi_{2}^{h}$ are normalized as follows:

$$
\begin{aligned}
\omega_{1}^{*} & :=\frac{\omega_{1}}{\pi^{2}}, \quad \xi_{1}^{\theta *}:=\frac{\xi_{1}^{\theta}}{\xi_{1}^{\mathrm{nq}}}=\frac{\xi_{1}^{\theta} \ell_{1}}{\pi}, \quad \xi_{1}^{h *}:=\frac{\xi_{1}^{h}}{\xi_{1}^{\mathrm{nq}}}=\frac{\xi_{1}^{h} \ell_{1}}{\pi} \\
\omega_{2}^{*} & :=\frac{\omega_{2}}{\pi^{2}}, \quad \xi_{2}^{\theta *}:=\frac{\xi_{2}^{\theta}}{\xi_{2}^{\mathrm{nq}}}=\frac{\xi_{2}^{\theta} \ell_{2}}{\pi}, \quad \xi_{2}^{h *}:=\frac{\xi_{2}^{h}}{\xi_{2}^{\mathrm{nq}}}=\frac{\xi_{2}^{h} \ell_{2}}{\pi} \\
\Rightarrow \lambda_{1} & :=\mathrm{e}^{\mathrm{i} \xi_{1}^{h} \ell_{1}}=\mathrm{e}^{\mathrm{i} \pi \xi_{1}^{h *}}, \quad \lambda_{2}:=\mathrm{e}^{\mathrm{i} \xi_{2}^{h} \ell_{2}}=\mathrm{e}^{\mathrm{i} \pi \xi_{2}^{h *}}
\end{aligned}
$$

where $\xi_{1}^{\mathrm{nq}}, \xi_{2}^{\mathrm{nq}}$ are the Nyquist frequencies of the discretization along the $2 \mathrm{D}$ axes. Using these normalized quantities, the characteristic equations of the continuous and discrete problems given by Equations (20) and (36) can be expressed as Equations (60) and (61), respectively.

$$
\begin{gathered}
\frac{\left(\xi_{1}^{\theta *}\right)^{2}}{\omega_{1}^{*}}+\frac{\left(\xi_{2}^{\theta *}\right)^{2}}{\omega_{2}^{*}}=1 \\
\left(\frac{\left[\left(1-\alpha_{1}\right)\left(\lambda_{2}^{2}+4 \lambda_{2}+1\right)+6 \alpha_{1} \lambda_{2}\right]\left(-1+2 \lambda_{1}-\lambda_{1}^{2}\right)}{6 \pi^{2} \omega_{1}^{*}}\right) \\
+\left(\frac{\left(-\lambda_{2}^{2}+2 \lambda_{2}-1\right)\left[\left(1-\alpha_{1}\right)\left(1+4 \lambda_{1}+\lambda_{1}^{2}\right)+6 \alpha_{1} \lambda_{1}\right]}{6 \pi^{2} \omega_{2}^{*}}\right) \\
-\left(\frac{\left[\left(1-\alpha_{2}\right)\left(\lambda_{2}^{2}+4 \lambda_{2}+1\right)\left(1+4 \lambda_{1}+\lambda_{1}^{2}\right)+36 \alpha_{2} \lambda_{2} \lambda_{1}\right]}{36}\right)=0
\end{gathered}
$$


For every choice of the pair $\left(\omega_{1}^{*}, \omega_{2}^{*}\right)$, the solution to Equation (60) will trace elliptic contours with the center at the origin in the $\xi_{1}^{*}-\xi_{2}^{*}$ plane. Owing to the inherent symmetry of the solutions, the dispersion plots are presented just in the first quadrant. Similar to $1 \mathrm{D}$, we require that the Nyquist frequencies of the discretization in $2 \mathrm{D}$ are always greater than the frequencies of the exact solution, i.e. $\min \left\{\xi_{1}^{\mathrm{nq}}, \xi_{2}^{\mathrm{nq}}\right\} \geqslant \xi_{o}$. Note that the following expressions are equivalent (三): $\min \left\{\xi_{1}^{\mathrm{nq}}, \xi_{2}^{\mathrm{nq}}\right\} \equiv \max \left\{\ell_{1}, \ell_{2}\right\} \equiv \max \left\{\omega_{1}^{*}, \omega_{2}^{*}\right\}$. Thus, restricting the domain to $\max \left\{\omega_{1}^{*}, \omega_{2}^{*}\right\} \in[0,1]$ guarantees this requirement:

$$
\omega^{*}:=\max \left\{\omega_{1}^{*}, \omega_{2}^{*}\right\} \in[0,1] \Leftrightarrow \min \left\{\xi_{1}^{\mathrm{nq}}, \xi_{2}^{\mathrm{nq}}\right\} \geqslant \xi_{o}
$$

Likewise, a mesh resolution of at least eight elements per wavelength is guaranteed by restricting the domain to $\omega^{*} \in\left[0, \frac{1}{16}\right]$. We study the following four cases concerned with the choice of the $\left(\alpha_{1}, \alpha_{2}\right)$ pair:

I: $\alpha_{1}=\alpha_{2}=\left(\frac{1}{2}\right)$. This case corresponds to the equation stencil associated with $\mathbf{A}^{0.5,0.5}:=$ $\left(\mathbf{A}^{\mathrm{fem}}+\mathbf{A}^{\mathrm{fdm}}\right) / 2$. Thus, the discrete system obtained here is the average of the systems obtained from the Galerkin FEM and the classical FDM. Recall that we can also obtain this stencil using the generalized Padé approximation in 2D and choosing the parameter $\gamma=2$.

II: $\alpha_{1}=\alpha_{2}=\alpha_{\theta}$ and $\theta=0$. This case corresponds to the $\alpha$-interpolation of the Galerkin FEM and the classical FDM. The local truncation error is zero along the direction $\theta=0$ whenever $\ell_{1}=\ell_{2}$.

III: $\alpha_{1}=\alpha_{2}=\alpha_{\theta}$ and $\theta=(\pi / 8)$. This case also corresponds to the $\alpha$-interpolation of the Galerkin FEM and the classical FDM. The local truncation error is zero along the direction $\theta=(\pi / 8)$ whenever $\ell_{1}=\ell_{2}$. Recall that choosing $\theta=(\pi / 8)$ leads to an optimized expression for the coefficient $r_{2}$.

IV: QSFEM, $\alpha_{1} \neq \alpha_{2} \neq 0$ and given by Equations (40) and (41). This case corresponds to the QSFEM presented in [15]. The local truncation error is zero along the directions $\theta=(\pi / 16)$ and $\theta=(3 \pi / 16)$ whenever $\ell_{1}=\ell_{2}$.

Note that for cases I, II and III, the relative phase and local truncation errors of the numerical solution diminish at a fourth-order rate i.e $O\left(\left(\xi_{o} \ell\right)^{4}\right)$ or equivalently $O\left(\omega^{2}\right)$. For the case IV, i.e the QSFEM, these errors diminish at a sixth-order rate i.e $O\left(\left(\xi_{o} \ell\right)^{6}\right)$ or equivalently $O\left(\omega^{3}\right)$.

In Figures 5 and 6, we plot the solutions to the characteristic equations of the continuous and discrete problems given by Equations (60) and (61), respectively. The contours of the continuous problem are drawn using the dashed line-style and the corresponding contour value displayed in a single text-box. Labeled solid line-style is used to display the contours of the discrete problem. Each figure is further divided into four sub-figures viz. (a)-(d) that correspond to the considered four cases I-IV. Within each sub-figure, the contours plots of the continuous and discrete problems are plotted and compared. In Figure 5, we plot the $\xi_{1}^{*}-\xi_{2}^{*}$ contours keeping $\omega_{1}^{*}=\omega_{2}^{*}$ i.e. $\ell_{1}=\ell_{2}$. The plotting domain considered here is $\left(\xi_{1}^{*}, \xi_{2}^{*}\right)=[0,0.55] \times[0,0.55]$. In Figure 6 , we plot the $\xi_{1}^{*}-\xi_{2}^{*}$ contours keeping $\omega_{2}^{*}=0.49 \omega_{1}^{*}$ i.e. $\ell_{2}=0.7 \ell_{2}$. The plotting domain considered here is again $\left(\xi_{1}^{*}, \xi_{2}^{*}\right)=[0,0.55] \times[0,0.55]$. In both the figures, contours are drawn for the values of $\omega^{*} \in\{(1 / 4),(1 / 9),(1 / 16),(1 / 25)\}$. These values of $\omega^{*}$ guarantee the presence of at least $4,6,8$ and 10 elements per wavelength, respectively. Note that except for the contour value $\omega^{*}=\left(\frac{1}{4}\right)$ in case I, the rest of the contours of the numerical solution are indistinguishable from their continuous counterparts. This is due to the fact that the relative local truncation error is of the order of $1 e-3$, which is small with respect to the scale of the plotting domain.

In order to quantify better the relative local truncation errors of the solutions, we compare them in Figure 7. This figure is further divided into four sub-figures viz. (a)-(d) that correspond to the considered four values of $\omega^{*}$, respectively, i.e. $\omega^{*} \in\{(1 / 4),(1 / 9),(1 / 16),(1 / 25)\}$. Within each sub-figure, the relative local truncation errors of the considered four cases viz. I-IV are plotted vs the direction $\beta$. Now we can clearly distinguish the errors related to the four cases. However in these figures the error associated with the case IV, i.e. the QSFEM, is indistinguishable from zero at this scale. In Figure 8 the relative local truncation errors of the solutions are plotted in the log-scale. The sub-figures are organized just like in Figure 7. Note that in Figures 7 and 8 

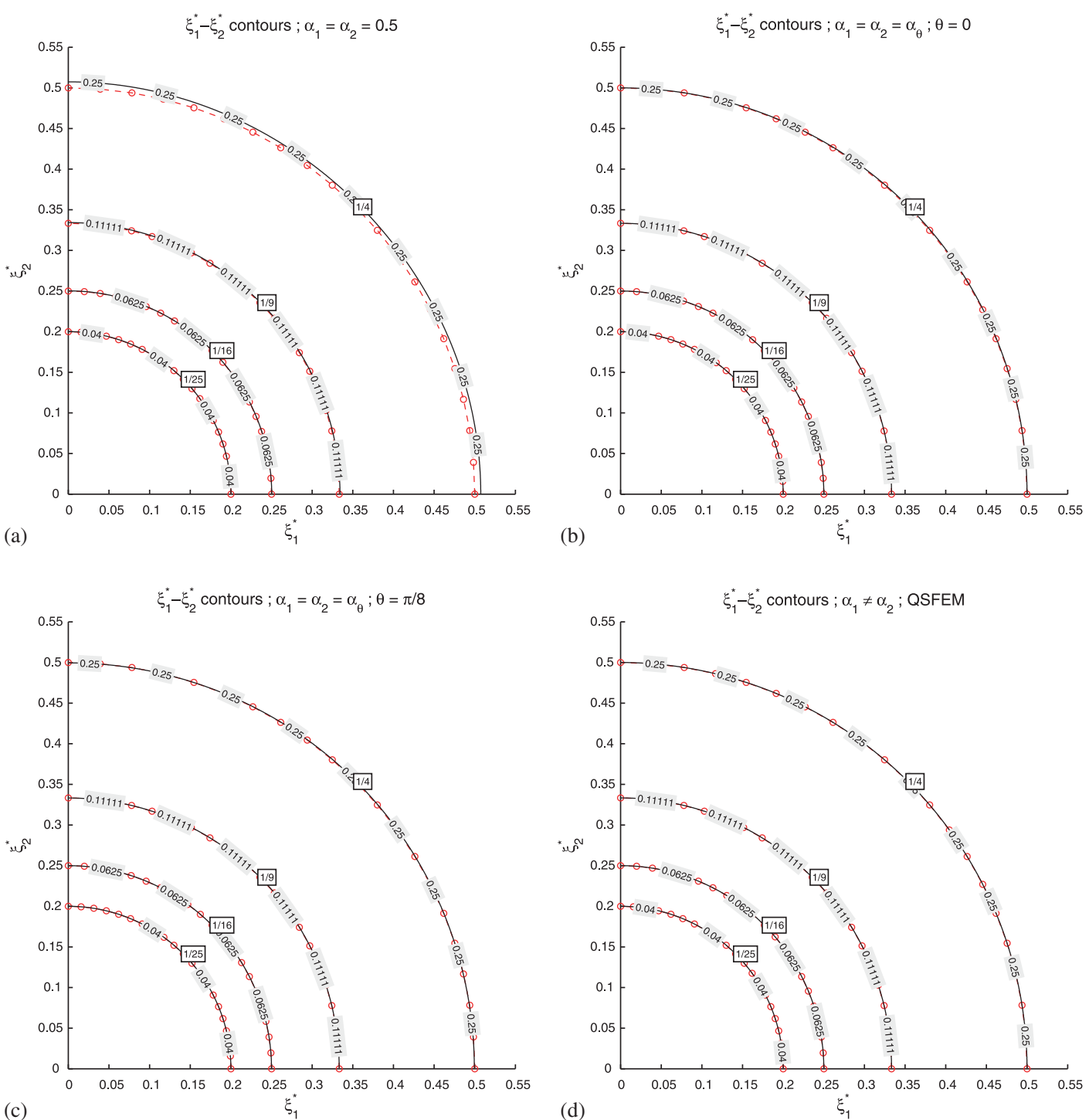

Figure 5. $\xi_{1}^{*}-\xi_{2}^{*}$ contours for $\omega^{*} \in\left\{\left(\frac{1}{4}\right),\left(\frac{1}{9}\right),\left(\frac{1}{16}\right),\left(\frac{1}{25}\right)\right\}$ and $\omega_{1}^{*}=\omega_{2}^{*}$. The dashed and solid line-styles correspond to the solutions of the continuous and discrete problems, respectively: (a) case I: $\alpha_{1}=\alpha_{2}=0.5$; (b) case II: $\alpha_{1}=\alpha_{2}=\alpha_{\theta}$ and $\theta=0$; (c) case III: $\alpha_{1}=\alpha_{2}=\alpha_{\theta}$ and $\theta=(\pi / 8)$; and (d) case IV: QSFEM, $\alpha_{1} \neq \alpha_{2} \neq 0$ and given by Equations (40) and (41).

the relative local truncation errors converge monotonically with respect to $\omega^{*}$, i.e. the plots of the errors with respect to the direction $\beta$ maintain their shape. This supports the conjuncture made in Section 4.5 that in the pre-asymptotic range, the location of the mini-max error is independent of $\omega^{*}$. Also we note that choosing $\theta=(\pi / 8)$ in the expression for $\alpha_{\theta}$, the maximum error is less than the one choosing $\theta=0$.

\subsection{Examples}

We consider the problem defined by Equation (1) $k \in\{1 \mathrm{e}-3,1 \mathrm{e}-4\}, s=1, f=0$ and the domain $\Omega=[0,1] \times[0,1]$. The Dirichlet boundary conditions are assigned such that the exact solution of Equation (1) is $\phi(\mathbf{x})=\sin \left(\xi^{\beta} \cdot \mathbf{x}\right)$, where $\beta$ is the chosen direction of wave propagation, $\xi^{\beta}:=$ $\xi_{o}(\cos (\beta), \sin (\beta))$ and $\xi_{o}:=\sqrt{s / k}$. Thus for the chosen values of $k$, the wavenumber $\xi_{o}$ takes the values in $\{10 \sqrt{10}, 100\}$. The following wave directions are considered: $\beta \in\{(\pi / 9),(\pi / 4)\}$. Seven uniform meshes $\left(\ell_{1}=\ell_{2}\right)$ of different resolutions are considered such that there are at least 4,6 , 

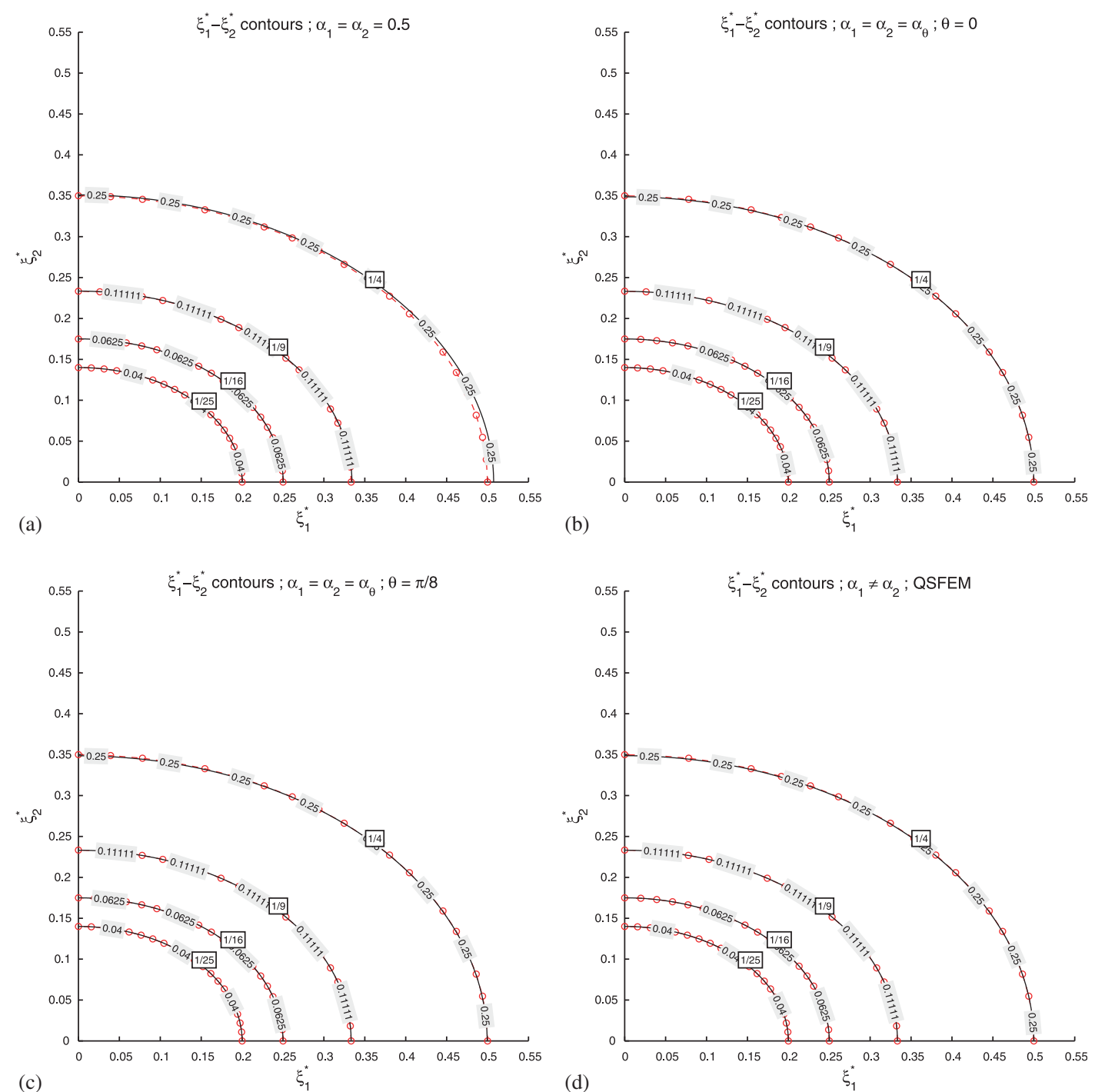

Figure 6. $\xi_{1}^{*}-\xi_{2}^{*}$ contours for $\omega^{*} \in\left\{\left(\frac{1}{4}\right),\left(\frac{1}{9}\right),\left(\frac{1}{16}\right),\left(\frac{1}{25}\right)\right\}$ and $\omega_{2}^{*}=0.49 \omega_{1}^{*}$. The dashed and solid line-styles correspond to the solutions of the continuous and discrete problems respectively: (a) case I: $\alpha_{1}=\alpha_{2}=0.5$; (b) case II: $\alpha_{1}=\alpha_{2}=\alpha_{\theta}$ and $\theta=0$; (c) case III: $\alpha_{1}=\alpha_{2}=\alpha_{\theta}$ and $\theta=(\pi / 8)$; and (d) case IV: QSFEM, $\alpha_{1} \neq \alpha_{2} \neq 0$ and given by Equations (40) and (41).

$8,10,12,14$ and 16 elements per wavelength, respectively. If the element length is chosen such that there are exactly $n$ elements per wavelength, then the value of $\xi^{*}=(2 / n)$ and $\omega^{*}=(2 / n)^{2}$. As it can be seen, all these meshes restrict the domain of $\omega^{*}$ to $\left[0, \frac{1}{4}\right]$. For these considerations, we study the convergence of the relative error in the following error norms:

$$
\begin{aligned}
& L^{2} \text { norm } \frac{\left\|\phi-\phi_{h}\right\|_{0}}{\|\phi\|_{0}}:=\frac{\left[\int_{\Omega}\left(\phi-\phi_{h}\right)^{2} \mathrm{~d} \Omega\right]^{1 / 2}}{\left[\int_{\Omega} \phi^{2} \mathrm{~d} \Omega\right]^{1 / 2}} \\
& H^{1} \text { semi-norm } \quad \frac{\left\|\phi-\phi_{h}\right\|_{1}}{\|\phi\|_{1}}:=\frac{\left[\int_{\Omega} \nabla\left(\phi-\phi_{h}\right) \cdot \nabla\left(\phi-\phi_{h}\right) \mathrm{d} \Omega\right]^{1 / 2}}{\left[\int_{\Omega} \nabla \phi \cdot \nabla \phi \mathrm{d} \Omega\right]^{1 / 2}} \\
& l^{\infty} \text { Euclidean norm } \frac{\left|\Phi_{e}-\Phi_{h}\right|_{\infty}}{\left|\Phi_{e}\right|_{\infty}}:=\frac{\max _{i}\left|\Phi_{e}^{i}-\Phi_{h}^{i}\right|}{\max _{i}\left|\Phi_{e}^{i}\right|}
\end{aligned}
$$



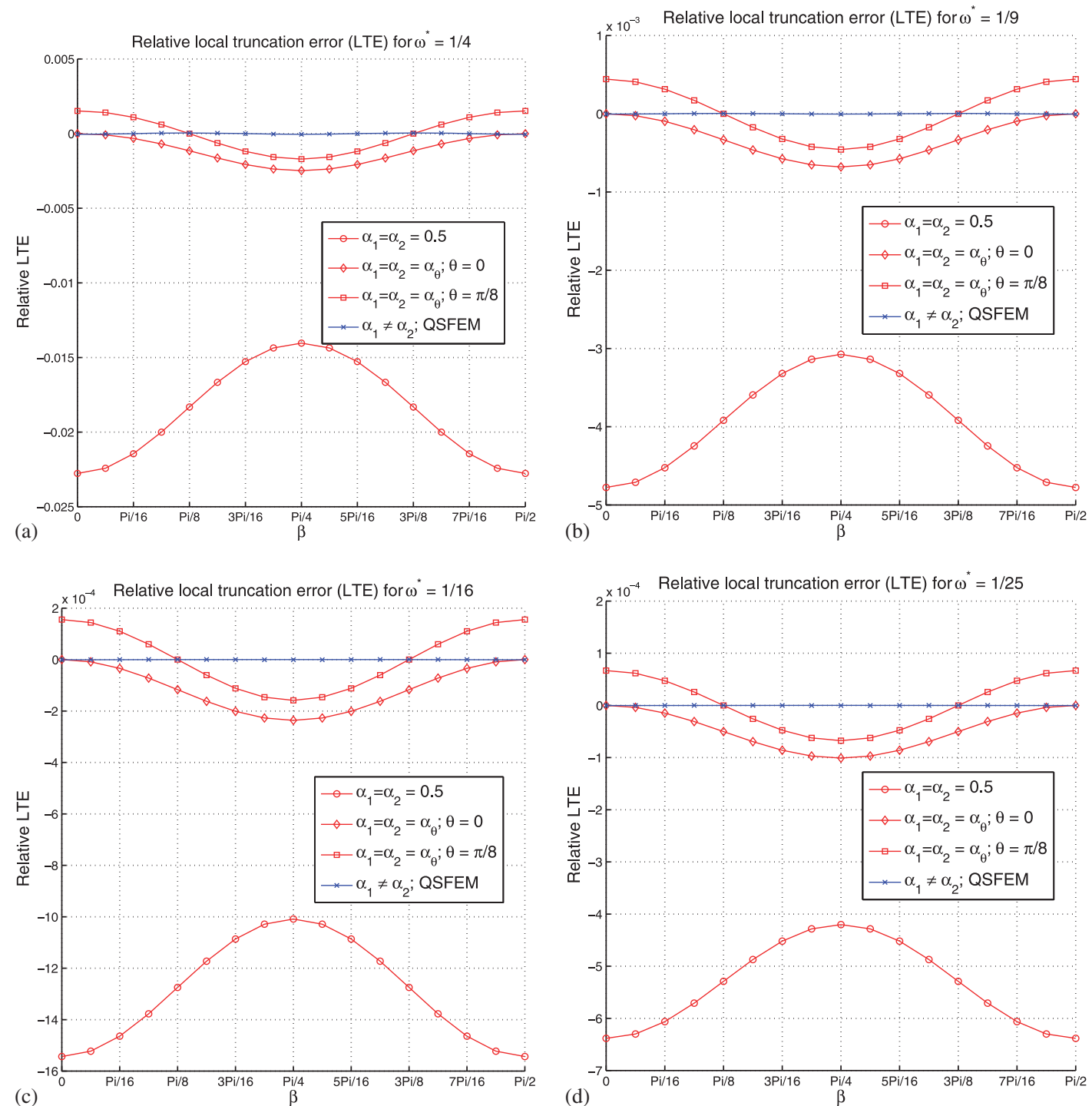

Figure 7. Relative local truncation error plots using $\ell_{1}=\ell_{2}$. Comparisons are made for the considered four cases viz. I-IV and for: (a) $\omega^{*}=\left(\frac{1}{4}\right)$; (b) $\omega^{*}=\left(\frac{1}{9}\right)$; (c) $\omega^{*}=\left(\frac{1}{16}\right)$; and (d) $\omega^{*}=\left(\frac{1}{25}\right)$.

In the convergence studies done here, the numerical solutions corresponding to the four cases, viz. I-IV, are compared with the following solutions: the nodally exact interpolant denoted by $I_{h} \phi$ and the best approximations with respect to the $L^{2}$ norm and the $H^{1}$ semi-norm denoted by $P_{h}^{0} \phi$ and $P_{h}^{1} \phi$, respectively. The solutions $I_{h} \phi, P_{h}^{0} \phi$ and $P_{h}^{1} \phi$ can be found as shown in Equation (64).

$$
\begin{aligned}
I_{h} \phi & :=N^{\mathrm{a}} \Phi_{e}^{\mathrm{a}} \\
\int_{\Omega} w_{h}\left(\phi-P_{h}^{0} \phi\right) \mathrm{d} \Omega & =0 \quad \forall w_{h} \in V_{0}^{h} \Rightarrow\left\|\phi-P_{h}^{0} \phi\right\|_{0} \leqslant\left\|\phi-\phi_{h}\right\|_{0} \quad \forall \phi_{h} \in V^{h} \\
\int_{\Omega} \nabla w_{h} \cdot \nabla\left(\phi-P_{h}^{1} \phi\right) \mathrm{d} \Omega & =0 \quad \forall w_{h} \in V_{0}^{h} \Rightarrow\left\|\phi-P_{h}^{1} \phi\right\|_{1} \leqslant\left\|\phi-\phi_{h}\right\|_{1} \forall \phi_{h} \in V^{h}
\end{aligned}
$$

As here the exact solution is sinusoidal, we have used a third-order Gauss quadrature rule to evaluate the expressions in Equations (63) and (64). Figures 9(a) and (b) illustrate the convergence of the relative error considering the wavenumber $\xi_{o}=10 \sqrt{10} \approx 31.62$, the wave direction $\beta=(\pi / 9)$ 

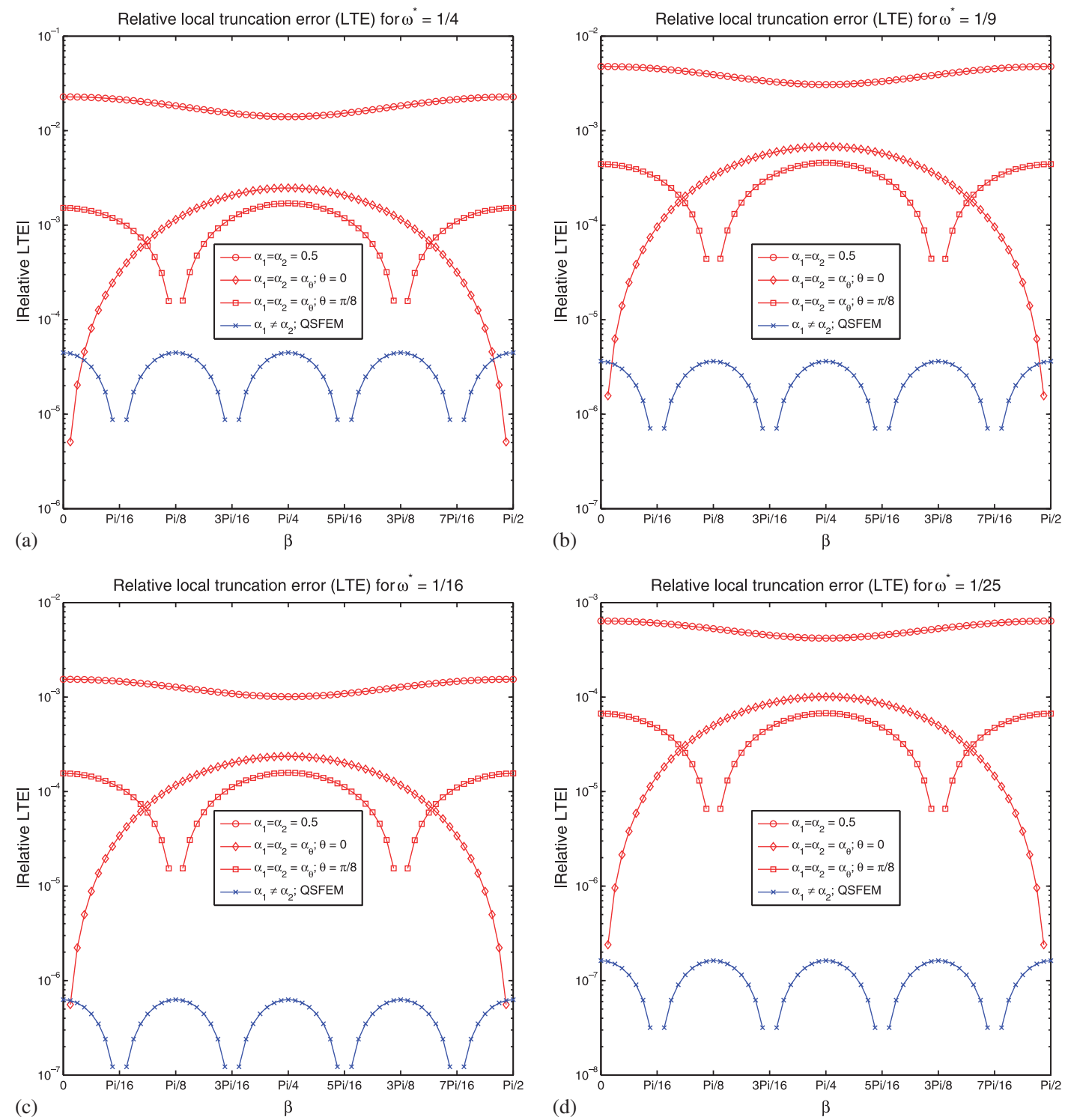

Figure 8. Log-scaled relative local truncation error plots using $\ell_{1}=\ell_{2}$. Comparisons are made for the considered four cases viz. I-IV and for: (a) $\omega^{*}=\left(\frac{1}{4}\right) ;\left(\right.$ b) $\omega^{*}=\left(\frac{1}{9}\right) ;\left(\right.$ c) $\omega^{*}=\left(\frac{1}{16}\right)$; and (d) $\omega^{*}=\left(\frac{1}{25}\right)$.

and using the $L^{2}$ norm and $H^{1}$ semi-norm, respectively. Figures 9(c) and (d) illustrate the same but now considering the wave direction $\beta=(\pi / 4)$. Clearly, the errors in the $L^{2}$ norm and $H^{1}$ seminorm corresponding to all the cases are greater than that of the respective best approximations. The error lines corresponding to the cases II-IV show a convergence trend indistinguishable from the error line of $I_{h} \phi$. On coarse meshes, the error line corresponding to case I deviates significantly from the error line of $I_{h} \phi$. Nevertheless, it quickly recovers the convergence trend of the later on finer meshes.

Figures 10(a) and (b) illustrate the convergence of the relative error considering the wavenumber $\xi_{o}=100$, the wave direction $\beta=(\pi / 9)$ and using the $L^{2}$ norm and $H^{1}$ semi-norm, respectively. Figures $10(\mathrm{c})$ and (d) illustrate the same but now considering the wave direction $\beta=(\pi / 4)$. Note that a higher value of $\xi_{o}$ introduces the 'pollution-effect' in the error lines as they deviate more from the error line of $I_{h} \phi$. However, the pollution effect is very small for cases II and III and is practically nil for case IV (sixth-order dispersion accuracy). 

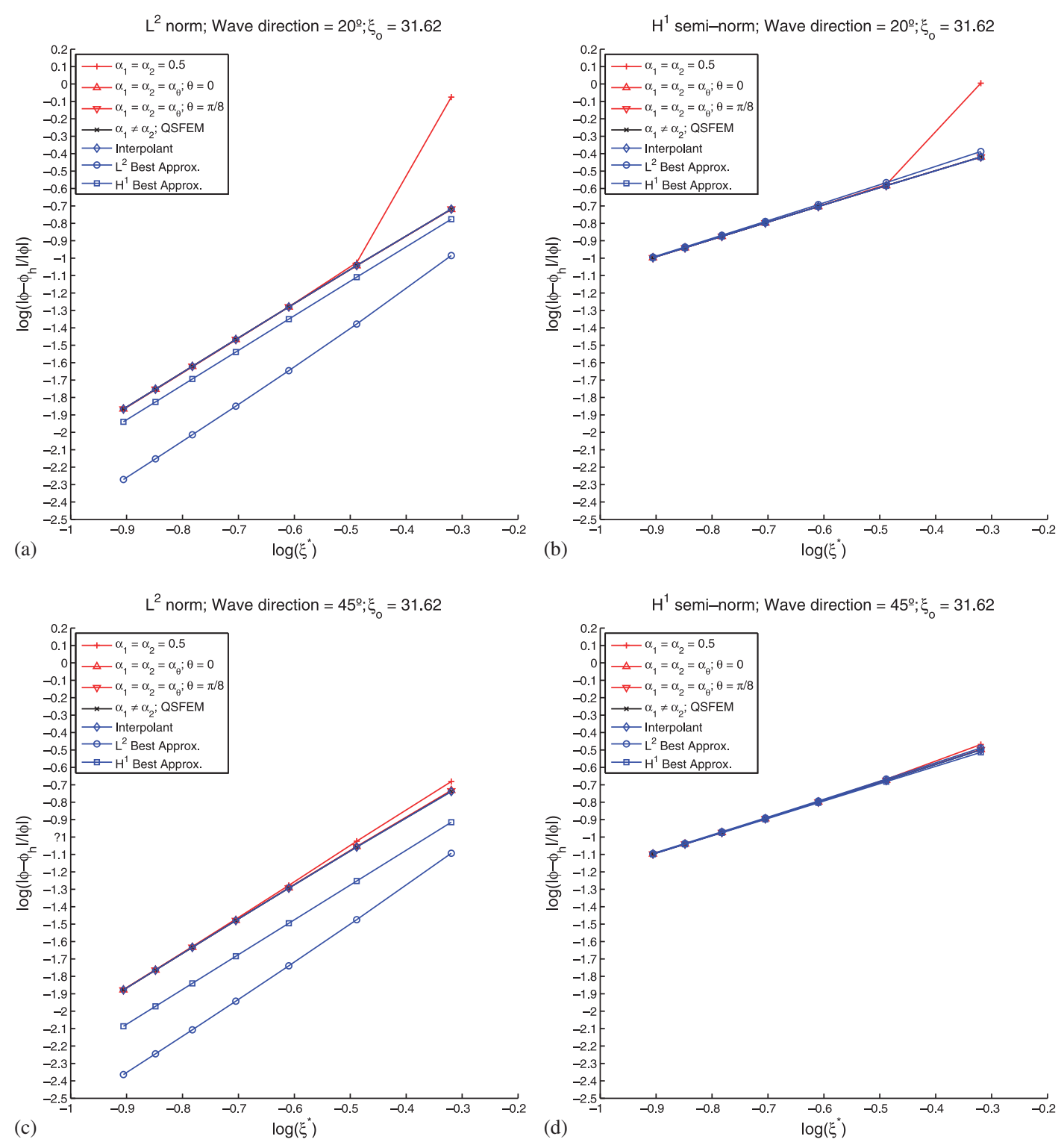

Figure 9. Convergence of the relative error considering $\xi_{0}=10 \sqrt{10}$ and for mesh resolutions that guarantee at least $n$ elements per wavelength, where $n \in\{4,6,8,10,12,14,16\}$. The considered norms and the wave directions are: (a) $L^{2}$ norm, $\beta=(\pi / 9)$; (b) $H^{1}$ semi-norm, $\beta=(\pi / 9)$; (c) $L^{2}$ norm, $\beta=(\pi / 4)$; and (d) $H^{1}$ semi-norm, $\beta=(\pi / 4)$.

Figure 11 illustrates the convergence of the relative error in the $l^{\infty}$ Euclidean norm. As a nodally exact solution requires that the dispersion error be zero, one may expect that the order of convergence in the $l^{\infty}$ Euclidean norm be the same as that of the corresponding dispersion error. In fact, the same is observed for the solutions corresponding to all the cases. The error lines of cases I-III converge at a fourth-order rate and that of case IV converges at a sixth-order rate. The error lines of the best approximations in the $L^{2}$ norm $\left(P_{h}^{0} \phi\right)$ and the $H^{1}$ semi-norm $\left(P_{h}^{1} \phi\right)$ converge at a second-order rate. The relative error of $P_{h}^{0} \phi$ is always greater that of $P_{h}^{1} \phi$. The pollution effect is now clearly visible for all the cases. Irrespective of the wave direction $\beta$, the error lines of all the cases shift higher with an increase in the wavenumber $\xi_{o}$. Meanwhile, the location of the error lines of $P_{h}^{0} \phi$ and $P_{h}^{1} \phi$ are practically unaffected by an increase in $\xi_{o}$ (no pollution). As the magnitudes of the relative error in the $l^{\infty}$ Euclidean norm for cases II-IV is 

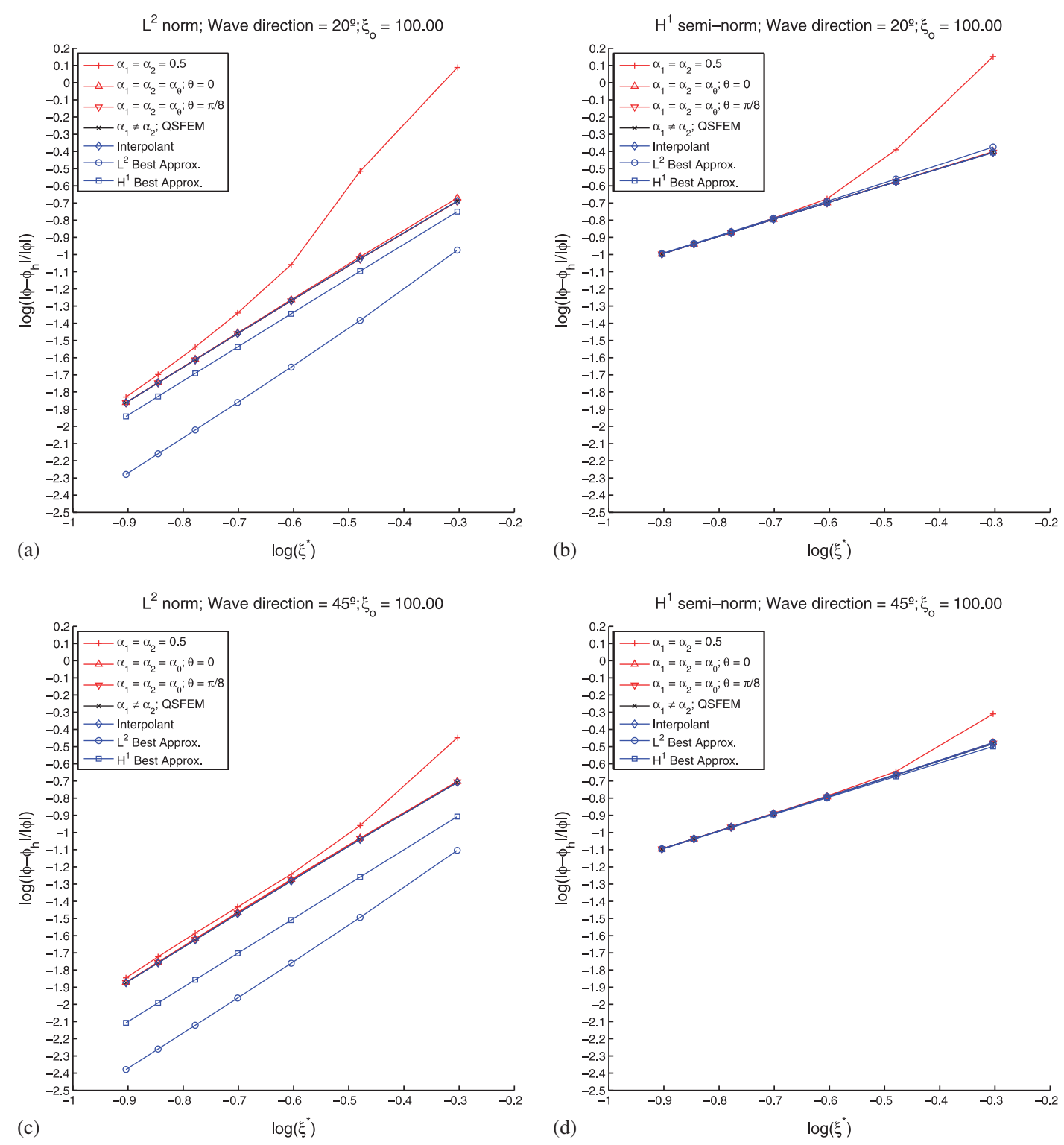

Figure 10. Convergence of the relative error considering $\xi_{o}=100$ and for mesh resolutions that guarantee at least $n$ elements per wavelength, where $n \in\{4,6,8,10,12,14,16\}$. The considered norms and the wave directions are: (a) $L^{2}$ norm, $\beta=(\pi / 9)$; (b) $H^{1}$ semi-norm, $\beta=(\pi / 9)$; (c) $L^{2}$ norm, $\beta=(\pi / 4)$; and (d) $H^{1}$ semi-norm, $\beta=(\pi / 4)$.

small (with respect to relative error of $I_{h} \phi$ in the $L^{2}$ norm and the $H^{1}$ semi-norm) for both the values of $\xi_{o}$, the pollution effect is hardly visible for these cases considering the relative error in the $L^{2}$ norm and the $H^{1}$ semi-norm.

Remark: As discussed in Section 3.4 and pointed out earlier in [6], although the discrete LBB constant in an average sense is inversely proportional to $\xi_{o}$, it has a more complicated behavior that tends its value to zero if $\xi_{o}$ approaches the zones of degeneracy (see Figure 2). Thus, pollution effects may be found not only for higher wavenumbers but also in those situations where the wavenumber $\xi_{o}$ approaches the zones of degeneracy. Of course, the higher the dispersion accuracy the closer the discrete eigenvalues to their continuous counterparts and narrower the zones of degeneracy. Also, if only the Dirichlet boundary conditions are prescribed (as is the case here), spurious amplitude and/or phase modulations might occur to satisfy them in spite of small 

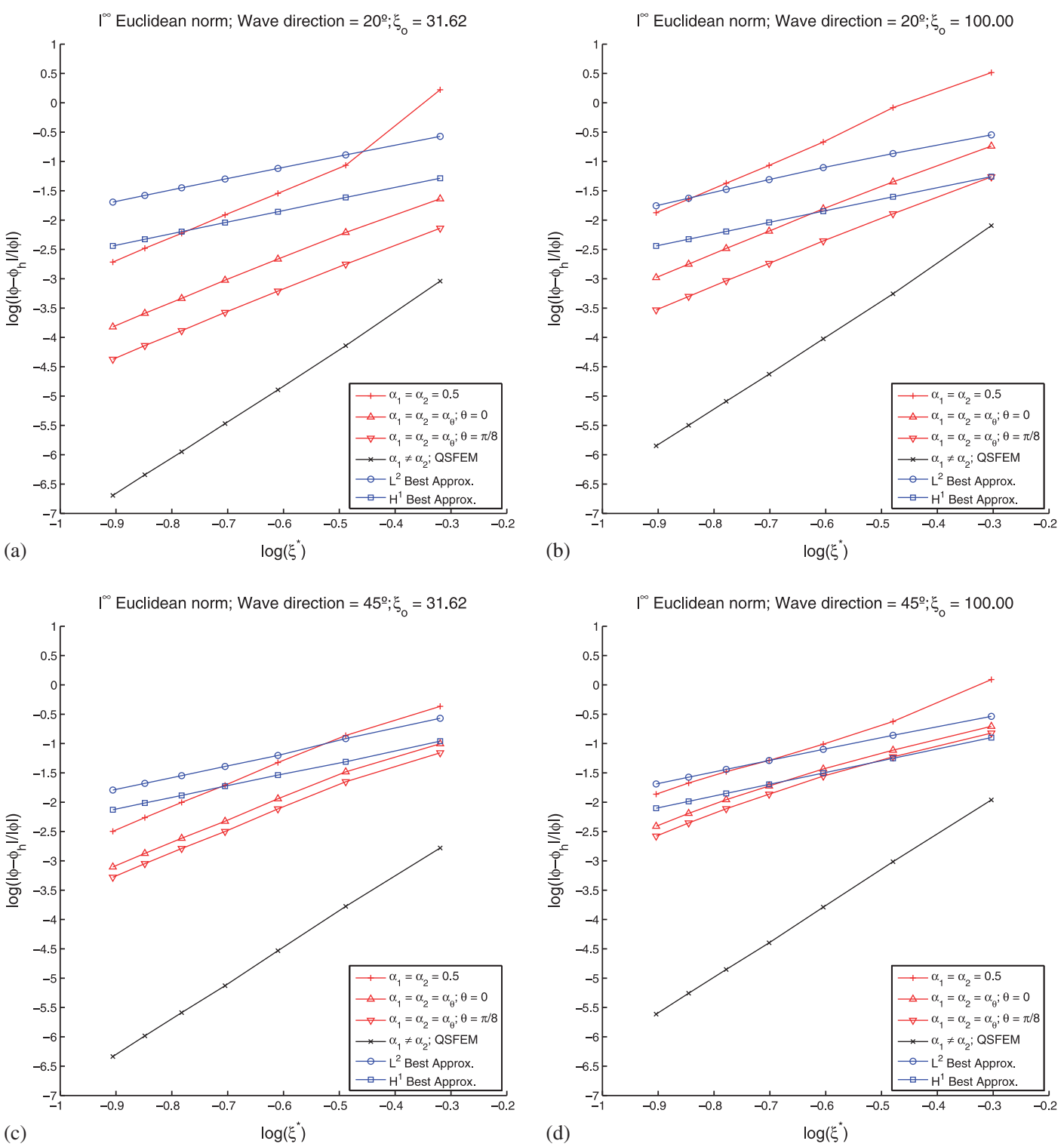

Figure 11. Convergence of the relative error in the $l^{\infty}$ Euclidean norm using: (a) $\xi_{o}=10 \sqrt{10}, \beta=(\pi / 9)$; (b) $\xi_{o}=100, \beta=(\pi / 9)$; (c) $\xi_{o}=10 \sqrt{10}, \beta=(\pi / 4)$; and (d) $\xi_{o}=100, \beta=(\pi / 4)$. The considered mesh resolutions guarantee at least $n$ elements per wavelength, where $n \in\{4,6,8,10,12,14,16\}$.

dispersion errors [11]. For the presented scheme, we have found vestiges of this behavior along the wave direction $\beta=0$.

\section{CONCLUSIONS AND OUTLOOK}

A fourth-order compact scheme on structured meshes is presented for the Helmholtz equation. The scheme consists of taking the $\alpha$-interpolation of the Galerkin FEM and the classical FDM. For the 2D analysis of this scheme, a generic nonstandard compact stencil involving two parameters $\alpha_{1}, \alpha_{2}$ is considered. In particular, this nonstandard compact stencil can model the aforementioned scheme (choosing $\alpha_{1}=\alpha_{2}=\alpha$ ) and also the QSFEM that has a dispersion accuracy of sixth-order. The expression for the numerical solution of this nonstandard stencil is given considering generic expressions for $\alpha_{1}, \alpha_{2}$ written as a series expansion in terms of $\omega:=\left(\xi_{o} \ell\right)^{2}$. Using this result, 
we provide the expressions for the phase and local truncation errors of this nonstandard compact stencil. In particular for our scheme, it is shown that these errors diminish at the rate $O\left(\left(\xi_{\rho} \ell\right)^{4}\right)$ or equivalently $O\left(\omega^{2}\right)$. An expression for the parameter $\alpha$ is given that minimizes the relative phase error in the pre-asymptotic range ( $\xi_{o} \ell$ small). Also, by this choice the local truncation error of the scheme along the direction $\beta=(\pi / 8)$ is made zero. Convergence studies of the relative error in the $L^{2}$ norm, the $H^{1}$ semi-norm and the $l^{\infty}$ Euclidean norm are done and the pollution effect is found to be small. In particular, using the optimal expression for $\alpha$ the relative error of our scheme in the $l^{\infty}$ Euclidean norm (for the considered examples and using at least 10 elements per wavelength) is found to be around or less than $1 \%$.

The abstractness in the definition of the QSFEM hinders its extension to unstructured meshes. This is a common problem faced by all the sixth-order methods proposed within the framework of the FDM. The recently proposed QOPG method addresses this issue and is able to attain a dispersion accuracy of the same order as the QSFEM on square meshes. Nevertheless, it uses a quadratic bubble perturbation function defined over a macro-element and the parameters multiplying these bubbles are found by solving local optimization problems involving a functional of the local truncation error. Alternate methods that achieve this objective were proposed earlier within a variational setting and with similar implementation/computational cost, viz. the RBFEM [26], the DGB method [32], the GPR method [34] etc. Can this path to obtain the QSFEM be simplified? That is the outlook of this paper.

Recall that the nonstandard compact stencil studied here has an additional structure that reduces its abstractness. This additional structure throws light on the extension of this stencil to unstructured meshes. In [70], a new Petrov-Galerkin method involving two parameters viz. $\alpha_{1}, \alpha_{2}$ is presented which yields this nonstandard compact stencil on rectangular meshes. Making the two parameters equal, i.e. $\alpha_{1}=\alpha_{2}=\alpha$, we recover the compact stencil obtained by the $\alpha$-interpolation of the Galerkin FEM and the classical central FDM. This Petrov-Galerkin method provides the counterparts of these two schemes on unstructured meshes and allows the treatment of natural boundary conditions (Neumann or Robin) and the source terms in a straightforward manner. This we believe would open door to design higher-order Petrov-Galerkin methods, which can be an alternative to the existing higher-order methods for the Helmholtz equation.

\section{ACKNOWLEDGEMENTS}

The first author acknowledges the economic support received through the FI pre-doctoral grant from the Department of Universities, Research and Information Society (Generalitat de Catalunya) and the European Social Fund. He also thanks Profs Ramon Codina and Carlos Felippa for many useful discussions. Support for this research provided by the projects SEDUREC of the Ministry of Science \& Education Spain, REALTIME from the European Research Council of the European Commission and AIDMAR of the Ministry of Science \& Innovation Spain are gratefully acknowledged.

\section{REFERENCES}

1. Courant R, Hilbert D. Methods of Mathematical Physics, vol. I. Wiley-Interscience: New York, 1989.

2. Chu-Hua S. The general derivation of Ritz method and Trefftz method in elastomechanics. Applied Mathematics and Mechanics 1982; 3(5):739-748.

3. Marin SP. A finite element method for problems involving the Helmholtz equation in two dimensional exterior regions. Thesis, Carnegie-Mellon University, Pittsburgh, PA, 1978.

4. Aziz AK, Werschulz A. On the numerical solution of Helmholtz's equation by the finite element method. SIAM Journal on Numerical Analysis 1980; 17(5):681-686.

5. Aziz AK, Kellogg RB, Stephens AB. A two point boundary value problem with a rapidly oscillating solution. Numerische Mathematik 1988; 53:107-121.

6. Demkowicz L. Asymptotic convergence in finite and boundary element methods, part 1: theoretical results. Computers and Mathematics with Applications 1994; 27(12):69-84.

7. Ihlenburg F, Babuska I. Finite element solution of the Helmholtz equation with high wave number part I: the h-version of the FEM. Computers and Mathematics with Applications 1995; 30(9):9-37.

8. Ihlenburg F, Babuska I. Finite element solution of the Helmholtz equation with high wave number part II: the h-p version of the FEM. SIAM Journal on Numerical Analysis 1997; 34(1):315-358. 
9. Babuska I, Sauter SA. Is the pollution effect of the FEM avoidable for the Helmholtz equation considering high wave numbers? SIAM Journal on Numerical Analysis 1997; 34(6):2392-2423.

10. Babuska IM, Sauter SA. Is the pollution effect of the FEM avoidable for the Helmholtz equation considering high wave numbers? SIAM Review 2000; 42(3):451-484.

11. Harari I, Hughes TJR. Finite element methods for the Helmholtz equation in an exterior domain: model problems. Computer Methods in Applied Mechanics and Engineering 1991; 87:59-96.

12. Thompson LL, Pinsky PM. A Galerkin least squares finite element method for the two-dimensional Helmholtz equation. International Journal for Numerical Methods in Engineering 1995; 38:371-397.

13. Harari I, Hughes TJR. Stabilized finite element methods for steady advection-diffusion with production. Computer Methods in Applied Mechanics and Engineering 1994; 115:165-191.

14. Babuska I, Osborn JE. Generalized finite element methods: their performance and their relation to mixed methods. SIAM Journal on Numerical Analysis 1983; 20(3):510-536.

15. Babuska I, Ihlenburg F, Paik ET, Sauter SA. A generalized finite element method for solving the Helmholtz equation in two dimensions with minimal pollution. Computer Methods in Applied Mechanics and Engineering 1995; 128:325-359.

16. Melenk JM, Babuska I. The partition of unity finite element method: basic theory and applications. Computer Methods in Applied Mechanics and Engineering 1996; 139:289-314.

17. Babuska I, Melenk JM. The partition of unity method. International Journal for Numerical Methods in Engineering 1997; 40:727-758.

18. Laghrouche O, Mohamed MS. Locally enriched finite elements for the Helmholtz equation in two dimensions. Computers and Structures 2008; DOI: 10.1016/j.compstruc.2008.04.006.

19. Franca LP, Farhat C, Macedo AP. Residual-free bubbles for the Helmholtz equation. International Journal for Numerical Methods in Engineering 1997; 40:4003-4009.

20. Barbone PE, Harari I. Nearly $H^{1}$-optimal finite element methods. Computer Methods in Applied Mechanics and Engineering 2000; 190(43-44):5679-5690.

21. Harari I, Gosteev K. Bubble-based stabilization for the Helmholtz equation. International Journal for Numerical Methods in Engineering 2007; 70:1241-1260.

22. Strouboulis T, Babuska I, Hidajat R. The generalized finite element method for Helmholtz equation: theory, computation, and open problems. Computer Methods in Applied Mechanics and Engineering 2006; 195:4711-4731.

23. Strouboulis T, Hidajat R, Babuska I. The generalized finite element method for Helmholtz equation. Part II: effect of choice of handbook functions, error due to absorbing boundary conditions and its assessment. Computer Methods in Applied Mechanics and Engineering 2008; 197:364-380.

24. Oberai AA, Pinsky PM. A multiscale finite element method for the Helmholtz equation. Computer Methods in Applied Mechanics and Engineering 1998; 154(3-4):281-297.

25. Cipolla JL. Subgrid modeling in a Galerkin method for the Helmholtz equation. Computer Methods in Applied Mechanics and Engineering 1999; 177:35-49.

26. Oberai AA, Pinsky PM. A residual-based finite element method for the Helmholtz equation. International Journal for Numerical Methods in Engineering 2000; 49:399-419.

27. Guasch O, Codina R. An algebraic subgrid scale finite element method for the convected Helmholtz equation in two dimensions with applications in aeroacoustics. Computer Methods in Applied Mechanics and Engineering 2007; 196:4672-4689.

28. Hauke G, Sangalli G, Doweidar MH. Combining adjoint stabilized methods for the advection-diffusion-reaction problem. Mathematical Models and Methods in Applied Sciences 2007; 17(2):305-326.

29. Farhat C, Harari I, Franca LP. The discontinuous enrichment method. Computer Methods in Applied Mechanics and Engineering 2001; 190:6455-6479.

30. Farhat C, Harari I, Hetmaniuk U. A discontinuous Galerkin method with Lagrange multipliers for the solution of Helmholtz problems in the mid-frequency regime. Computer Methods in Applied Mechanics and Engineering 2003; 192:1389-1419.

31. Alvarez GB, Loula AFD, Dutra do Carmo EG, Rochinha FA. A discontinuous finite element formulation for Helmholtz equation. Computer Methods in Applied Mechanics and Engineering 2006; 195:4018-4035.

32. Loula AFD, Alvarez GB, Dutra do Carmo EG, Rochinha FA. A discontinuous finite element method at element level for Helmholtz equation. Computer Methods in Applied Mechanics and Engineering 2007; 196:867-878.

33. Felippa CA, Oñate E. Nodally exact Ritz discretizations of 1D diffusion-absorption and Helmholtz equations by variational FIC and modified equation methods. Computational Mechanics 2007; 39(2):91-111.

34. Dutra do Carmo EG, Alvarez GB, Loula AFD, Rochinha FA. A nearly optimal Galerkin projected residual finite element method for Helmholtz problem. Computer Methods in Applied Mechanics and Engineering 2008; 197:1362-1375.

35. Harari I. A survey of finite element methods for time-harmonic acoustics. Computer Methods in Applied Mechanics and Engineering 2006; 195:1594-1607.

36. Loula AFD, Fernandes DT. A quasi optimal Petrov-Galerkin method for Helmholtz problem. International Journal for Numerical Methods in Engineering 2009; 80:1595-1622.

37. Fernandes DT, Loula AFD. Quasi optimal finite difference method for Helmholtz problem on unstructured grids. International Journal for Numerical Methods in Engineering 2010; 82:1244-1281.

38. Harari I, Turkel E. Accurate finite difference methods for the time-harmonic wave propagation. Journal of Computational Physics 1995; 119:252-270.

Copyright (C) 2010 John Wiley \& Sons, Ltd.

Int. J. Numer. Meth. Engng 2011; 86:18-46

DOI: $10.1002 / \mathrm{nme}$ 
39. Singer I, Turkel E. High-order finite difference methods for the Helmholtz equation. Computer Methods in Applied Mechanics and Engineering 1998; 163:343-358.

40. Sheu TWH, Chen CF, Hsieh LW. A highly accurate Helmholtz scheme for modeling scattering wave. Computer Methods in Applied Mechanics and Engineering 2004; 193:5573-5583.

41. Lambe L, Luczak R, Nehrbass J. A new finite difference method for the Helmholtz equation using symbolic computation. International Journal of Computational Engineering Science 2003; 4(1):121-144.

42. Tsukerman I. A class of difference schemes with flexible local approximation. Journal of Computational Physics 2006; 211:659-699.

43. Sutmann G. Compact finite difference schemes of sixth order for the Helmholtz equation. Journal of Computational and Applied Mathematics 2007; 203:15-31.

44. Singer I, Turkel E. Sixth order accurate finite difference schemes for the Helmholtz equation. Journal of Computational Acoustics 2006; 14(3):339-351.

45. Nabavi M, Kamran Siddiqui MH, Dargahi J. A new 9-point sixth-order accurate compact finite difference method for the Helmholtz equation. Journal of Sound and Vibration 2007; 307:972-982.

46. Bellman R, Kashef BG, Kasti J. Differential quadrature: a technique for the rapid solution of nonlinear partial differential equations. Journal of Computational Physics 1972; 10:40-52.

47. Shu C, Xue H. Solution of Helmholtz equation by differential quadrature method. Computer Methods in Applied Mechanics and Engineering 1999; 175:203-212.

48. Rose ME. Weak-element approximations to elliptic differential equations. Numerische Mathematik 1975; 24:185-204.

49. Jirousek J. Basis for development of large finite elements locally satisfying all field equations. Computer Methods in Applied Mechanics and Engineering 1978; 14:65-92.

50. Herrera I. Trefftz method. In Topics in Boundary Element Research, Volume 1: Basic Principles and Applications, Chapter 10, Brebbia CA (ed.). Springer: New York, 1984; 230-258.

51. Zieliński AP, Zienkiewicz OC. Generalized finite element analysis with T-complete boundary solution functions. International Journal for Numerical Methods in Engineering 1985; 21:509-528.

52. Jirousek J, Zieliński AP. Survey of Trefftz-type element formulations. Computers and Structures 1997; 63(2): 225-242.

53. Herrera I. Trefftz method: a general theory. Numerical Methods for Partial Differential Equations 2000; 16(6): 561-580.

54. Goldstein CI. The weak element method applied to Helmholtz type equations. Applied Numerical Mathematics 1986; 2(3-5):409-426.

55. Stojek M. Finite T-elements for the Poisson and Helmholtz equations. Thesis, École Polytechnique Fédérale de Lausanne, Lausanne, 1996.

56. Cessenat O, Despres B. Application of an ultra weak variational formulation of elliptic PDES to the twodimensional Helmholtz problem. SIAM Journal on Numerical Analysis 1998; 35(1):255-299.

57. Monk P, Wang DQ. A least-squares method for the Helmholtz equation. Computer Methods in Applied Mechanics and Engineering 1999; 175:121-136.

58. Li ZC. The Trefftz method for the Helmholtz equation with degeneracy. Applied Numerical Mathematics 2008; 58:131-159.

59. Goudreau GL. Evaluation of discrete methods for the linear dynamic response of elastic and viscoelastic solids. Ph.D. Thesis, Department of Civil Engineering, University of California, Berkeley, CA, 1970.

60. Goudreau GL, Taylor RL. Evaluation of numerical integration methods in elastodynamics. Computer Methods in Applied Mechanics and Engineering 1972; 2:69-97.

61. Ishihara K. Convergence of the Finite Element Method Applied to the Eigenvalue Problem $\Delta u+\lambda u=0$, vol. 13. Publications of the Research Institute for Mathematical Sciences: Kyoto University, 1977; 47-60.

62. Niki H, Sawami H, Ikeuchi M, Okamoto N. The alpha interpolation method for the solution of an eigenvalue problem. Journal of Computational and Applied Mathematics 1982; 8(1):15-19.

63. Ikeuchi M, Inoue K, Sawami H, Niki H. Arbitrarily shaped hollow waveguide analysis by the $\alpha$-interpolation method. SIAM Journal on Applied Mathematics 1981; 40(1):90-98.

64. Nakamura M, Hirasawa M. Eigenvalues of the Schrodinger equation by the $\alpha$-interpolation method. SIAM Journal on Applied Mathematics 1983; 43(6):1286-1293.

65. Trefethen LN. Group velocities in finite difference schemes. SIAM Review 1982; 24(2):113-136.

66. Collatz L. The Numerical Treatment of Differential Equations (3rd edn). Springer: Berlin, 1960.

67. Vichnevetsky R, Bowles JB. Fourier Analysis of Numerical Approximations of Hyperbolic Equations. SIAM Studies in Applied Mathematics, vol. 5. SIAM: Philadelphia, PA, 1982.

68. Johns PB, Beurle RL. Numerical solution of two-dimensional scattering problems using a transmission line matrix. Proceedings of the IEE 1971; 118(12):1203-1208.

69. Simons NRS, Sebak AA. Spatially weighted numerical models for the two-dimensional wave equation: FD algorithm and synthesis of the equivalent TLM model. International Journal of Numerical Modelling: Electronic Networks, Devices and Fields 1993; 6:47-65.

70. Nadukandi P, Oñate E, Garcia J. A Petrov-Galerkin formulation for the alpha-interpolation of FEM and FDM stencils. Applications to the Helmholtz equation. International Journal for Numerical Methods in Engineering 2010; submitted. 